\title{
A general principle of dendritic constancy - a neuron's size and shape invariant excitability
}

*Hermann Cuntz ${ }^{\mathrm{a}, \mathrm{b}}$, Alexander D Bird ${ }^{\mathrm{a}, \mathrm{b}}$, Marcel Beining ${ }^{\mathrm{a}, \mathrm{b}, \mathrm{c}, \mathrm{d}}$, Marius Schneider ${ }^{\mathrm{a}, \mathrm{b}}$, Laura Mediavilla $^{\mathrm{a}, \mathrm{b}}$, Felix Z Hoffmann ${ }^{\mathrm{a}, \mathrm{b}}$, Thomas Deller ${ }^{\mathrm{c}, 1}$, Peter Jedlicka ${ }^{\mathrm{b}, \mathrm{c}, \mathrm{e}, 1}$

${ }^{a}$ Ernst Strüngmann Institute (ESI) for Neuroscience in cooperation with the Max Planck Society, 60528 Frankfurt am Main, Germany

${ }^{b}$ Frankfurt Institute for Advanced Studies, 60438 Frankfurt am Main, Germany

${ }^{c}$ Institute of Clinical Neuroanatomy, Neuroscience Center, Goethe University, 60590

Frankfurt am Main, Germany

${ }^{d}$ Max Planck Insitute for Brain Research, 60438 Frankfurt am Main, Germany

${ }^{e}$ ICAR3R - Interdisciplinary Centre for 3Rs in Animal Research, Justus Liebig University

Giessen, 35390 Giessen, Germany

${ }^{1}$ Joint senior authors

*cuntz@fias.uni-frankfurt.de

\section{Keywords}

Electrotonic analysis, Compartmental model, Morphological model, Excitability, Neuronal scaling, Passive normalisation, Cable theory 


\section{In brief}

We show that realistic neuron models essentially collapse to point neurons when stimulated by randomly distributed inputs instead of by single synapses or current injection in the soma.

\section{Highlights}

- A simple equation that predicts voltage in response to distributed synaptic inputs.

- Responses to distributed and clustered inputs are largely independent of dendritic length.

- Spike rates in various Hodgkin Huxley $(\mathrm{HH})$ like or Leaky Integrate-and-Fire (LIF) models are largely independent of morphology.

- Precise spike timing (firing pattern) depends on dendritic morphology.

- NeuroMorpho.Org database-wide analysis of the relation between dendritic morphology and electrophysiology.

- Our equations set precise input-output relations in realistic dendrite models. 


\section{Abstract}

Reducing neuronal size results in less cell membrane and therefore lower input conductance. Smaller neurons are thus more excitable as seen in their voltage responses to current injections in the soma. However, the impact of a neuron's size and shape on its voltage responses to synaptic activation in dendrites is much less understood. Here we use analytical cable theory to predict voltage responses to distributed synaptic inputs and show that these are entirely independent of dendritic length. For a given synaptic density, a neuron's response depends only on the average dendritic diameter and its intrinsic conductivity. These results remain true for the entire range of possible dendritic morphologies irrespective of any particular arborisation complexity. Also, spiking models result in morphology invariant numbers of action potentials that encode the percentage of active synapses. Interestingly, in contrast to spike rate, spike times do depend on dendrite morphology. In summary, a neuron's excitability in response to synaptic inputs is not affected by total dendrite length. It rather provides a homeostatic input-output relation that specialised synapse distributions, local non-linearities in the dendrites and synaptic plasticity can modulate. Our work reveals a new fundamental principle of dendritic constancy that has consequences for the overall computation in neural circuits.

\section{Introduction}

Because of their cell-type specific characteristic morphologies, dendritic trees have commonly 19 been assumed to be crucial for a neuron's intrinsic computations. It has been shown that altering the morphology (Mainen and Sejnowski, 1996; Vetter et al., 2001) or the topology (van Elburg and van Ooyen, 2010; van Ooyen et al., 2002) of neurons while keeping the electrotonic features unchanged has a profound impact on the spiking behaviour of the cell. On the other hand, the morphology of dendrites has been shown to be largely predicted by connectivity rules (Cuntz et al. 2010) rather than by the specific computation that they perform. Also, dendrites were shown to follow general principles that equalise passive (Bird and Cuntz, 2016; Cuntz et al., 2007, Connelly et al., 2016; Jaffe and Carnevale, 1999) and active (Häusser, 2001: Magee, 2000) signal propagation indicating that the blueprints of computation for single neurons might be more stereotypical than previously assumed. In fact, principles of conservative scaling that preserve electrotonic features have been proposed for a number of cell types (Bakken and Stevens, 2011; Bekkers and Stevens, 1990: Cuntz et al., 2013). Similarly, 
general morphological scaling laws, have been discovered for dendritic arbours of various sizes (Cuntz et al., 2012; Snider et al., 2010; Teeter and Stevens, 2011). However, it remains unclear how electrotonic and morphological scaling principles relate to one another and how their interplay would affect well-known neuronal computations in dendrites (e.g. Branco et al., 2010; Gabbiani et al., 2002; Poirazi et al., 2003b a; Single and Borst, 1998). Therefore, we study here the dependence of input-output properties of dendrites on their size and shape.

One of the most eminent electrophysiological features of neurons that depend on dendritic shape is the input conductance (Koch et al., 1990; Rall et al., 1967). Smaller cells with smaller input conductances are more excitable for somatic current injections than larger cells because a 40 voltage threshold for spike initiation is reached with a lower input current in accordance with Ohm's law (Chavlis et al. 2017: Šišková et al. 2014). This relation is true for somatic activation 41 of neurons and its size- and shape-dependence of voltage responses is well understood. 42 However, the corresponding effect of changes in input conductance on voltage responses to distributed synaptic inputs have not been sufficiently studied. Rules identified for current transfer within dendritic arbours (Bird and Cuntz, 2016; Cuntz et al., 2007; London et al., 1999; Rall and Rinzel, 1973; Rinzel and Rall, 1974) have allowed the prediction of responses to individual or a few synaptic inputs (Magee, 2000; Williams and Stuart, 2003). Similar rules should be applicable at the level of richer synaptic input but they have not yet been identified. In this work, we specifically address the question how neuronal firing rate and firing patterns are affected by dendritic size and shape in the case of multiple, distributed synaptic inputs. We show that passive electrotonic principles generally render the synaptically driven excitability of neurons invariable to length for the entire range of existing dendritic trees. Since this dendritic constancy principle supports the stability of neuronal spiking, it may complement other well-established synaptic and intrinsic mechanisms of firing rate homeostasis (Turrigiano and Nelson, 2004). 

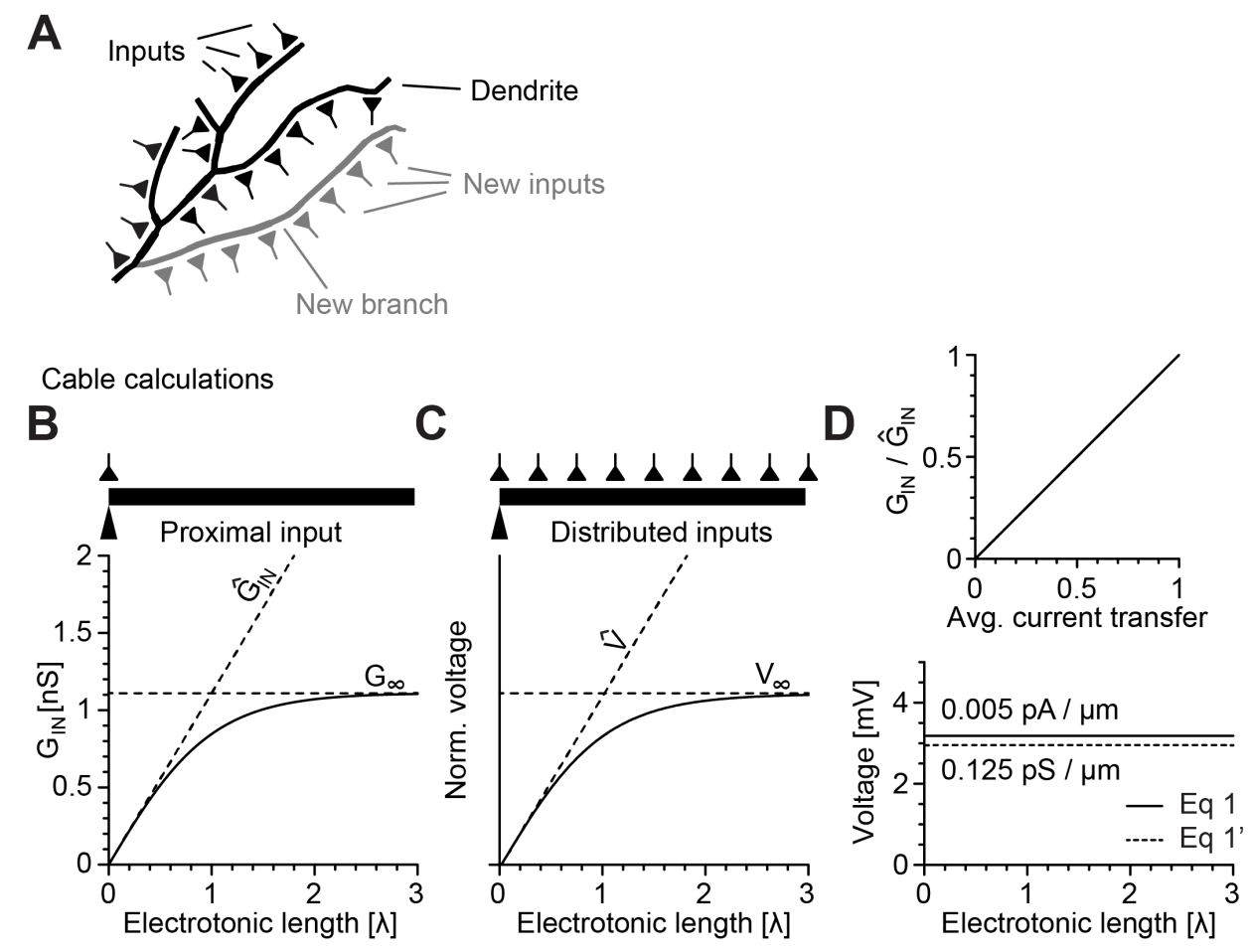

Fig 1. Analytical prediction indicates that responses to distributed synaptic inputs in a cable are independent of cable length.

A, Sketch illustrating the impact of a new branch on dendrite length and number of synapses. B, Input conductance $G_{I N}$ of cables with constant diameters for a wide range of electrotonic lengths. $G_{\infty}$, the input conductance of a semi-infinite cable and $\hat{G}_{I N}$, the collapsed total membrane conductance are indicated by dashed lines for reference. C, Mean steady-state voltage responses to distributed inputs as a function of electrotonic length. $V_{\infty}$, the response to distributed synapses in the semi-infinite cable and $\hat{V}$, the linear extrapolation of the voltage response at the root and therefore the response in a collapsed cable are indicated by dashed lines. D, Bottom panel shows steady-state voltage responses at the proximal end to distributed current injections every $\mu \mathrm{m}$ (straight line, Equation 1 ) and to synaptic inputs every $\mu \mathrm{m}$ (dashed line, Equation $\mathbf{1}^{\prime}$ ). Top panel shows the average current transfer versus the ratio of $G_{I N}$ to $\hat{G}_{I N}$ for the cables of varying lengths from $\mathbf{B}$. Panels $\mathbf{B}-\mathbf{D}$ were obtained from numerical simulations validating exactly the results of our analytical calculations.

\section{Results}

The idea behind this work comes from the simple reasoning that while larger neurons are $\quad 58$ in principle less excitable they also receive more synapses (Figure 1 A). The higher input 59 conductance and resulting decreased excitability might therefore compensate for the increase 60 of effective current the neuron receives through its synaptic inputs. In contrast to most 61 traditional theoretical studies on dendritic integration with their focus on somatic activation 62 
of the cell or activation with few synapses, we therefore focus here on the voltage responses to 63 distributed synaptic inputs. In the following, we first study these relations analytically in the 64 simple passive cable and subsequently move to passive and then active responses in dendritic 65 trees with their full morphology.

\section{Analytical calculations for passive cables predict length-invariant responses 67 to distributed synaptic inputs

Experimentally, the input conductance that predicts a neuron's excitability is most typically using Ohm's law to relate conductance, current and voltage. The corresponding analytical calculations for a simple dendritic cable are readily available from classical cable theory introduced to neuroscience by Wilfrid Rall. Considerations of current spread in a passive cylinder allow one to predict the input conductance $G_{I N}$ for any cable of electrotonic length $L$ measured in terms of $\lambda=\sqrt{\frac{G_{i} d}{4 G_{m}}}$, the electrotonic length constant, a distance unit over which the voltage decays to about a third of the proximal voltage (Koch and Segev, 1999; Rushton, 1937). Here, the diameter is $d$, the specific axial conductance is $G_{i}$ and the specific membrane conductance is $G_{m}$. For short cables, $G_{I N}$ increases nearly linearly with $L$ as it approximates the collapsed input conductance $\hat{G}_{I N}=G_{m} \pi d \lambda L$ of the cable, the total sum of the membrane leak (Figure 1B). At the other extreme, $G_{I N}$ at the proximal sealed end in a semi-infinite cable is $G_{\infty}=G_{m} \pi d \lambda$, the total conductance of a $\lambda$ length cylinder since $\hat{G}_{I N}=G_{\infty} L$. For longer cables of electrotonic length $L$, the input conductance at the proximal end $G_{I N}$ approaches $G_{\infty}$ asymptotically as $G_{I N}=G_{\infty} \tanh (L)$ (Figure 1B). More distal patches of membrane therefore contribute less and less to the total proximal input conductance, setting with $G_{\infty}$ a lower bound for the overall excitability of the cell. In all cases, increasing either the diameter $d$, the specific axial conductance $G_{i}$ or the specific membrane conductance $G_{m}$ all increase the input conductance as well as $G_{\infty}$.

As mentioned earlier, apart from their larger input conductance, larger cells also receive more synaptic inputs if one considers constant synaptic density. In the case of very short cables, 89 the increase in input conductance is intuitively perfectly compensated by the increase in 90 number of synapses since both scale linearly with $L$. Interestingly, however, the impact of 91 distal synapses onto voltage at the proximal end diminishes with distance in the very same 92 way as the impact of a distal patch of membrane on the proximal input conductance. The 93 
reference values $V_{\infty}$, the average proximal voltage in response to distributed synapses over the 94 dendritic length of a semi-infinite cable, and $\hat{V}$, the voltage response of these synapses when 95 they are collapsed to an isopotential piece of membrane, behave similarly to their respective 96 input conductance counterparts $G_{\infty}$ and $\hat{G}_{I N}$ (Figure 1C). It can be shown analytically (see ${ }_{97}$ Methods, "Cable equation for responses to distributed inputs", Equations 3-77) that, along 98 the entire electrotonic length, input conductance and synaptic currents cancel one another 99 precisely. Correspondingly, the average current transfer throughout the cable, i.e. the fraction of injected synaptic current that reaches the proximal cable end, is equal to the ratio of $G_{I N}$ to $\hat{G}_{I N}$, i.e. the fraction of overall conductance felt at the proximal cable end (Figure 10 , top panel).

The voltage responses to distributed current injections $I_{\text {dist }}$ per unit length are therefore equivalent to the total current injected over the entire metric length $l=\lambda L$ of the neuron into its collapsed membrane leak, i.e.

$$
V_{d i s t}=\frac{I_{d i s t} l}{\hat{G}_{I N}}=\frac{I_{d i s t}}{G_{m} \pi d}
$$

(Figure 1D, bottom panel, straight line). From this particular application of Ohm's law to dendritic trees, the voltage response to distributed inputs is entirely independent of neuronal cable length while it depends only on the specific conductance per surface membrane $G_{m}$ and the diameter $d$ of the cable as well as $G_{i}$, since $I_{d i s t}$ is defined per unit electrotonic length. This is in stark contrast with voltage responses to proximal "somatic" current injections where $V=\frac{I}{\left.G_{\infty} \tanh (L)\right)}$, and length impacts the excitability of a neuron by decreasing $V$ dramatically when increasing $L$. It is clear, however, that in a realistic setting, excitatory neuronal inputs produce synaptic currents that are distributed over the dendritic tree rather than being somatic. In fact, synaptic currents flow through synaptic conductances that further increase the overall conductance per unit length assuming that synaptic densities are homogeneous and constant. The corresponding voltage responses to distributed synaptic conductances are therefore slightly lower than the ones from current injections. However, also these effects remain independent of total cable length:

$$
V_{s y n}=\frac{I_{\text {syn }}}{G_{m} \pi d+G_{s y n}}
$$

(Figure 1D, dashed line). In conclusion, our analytical calculations and numerical simula- 120 tions reveal a new electrotonic principle of dendritic constancy ensuring an equal impact of ${ }_{121}$ 

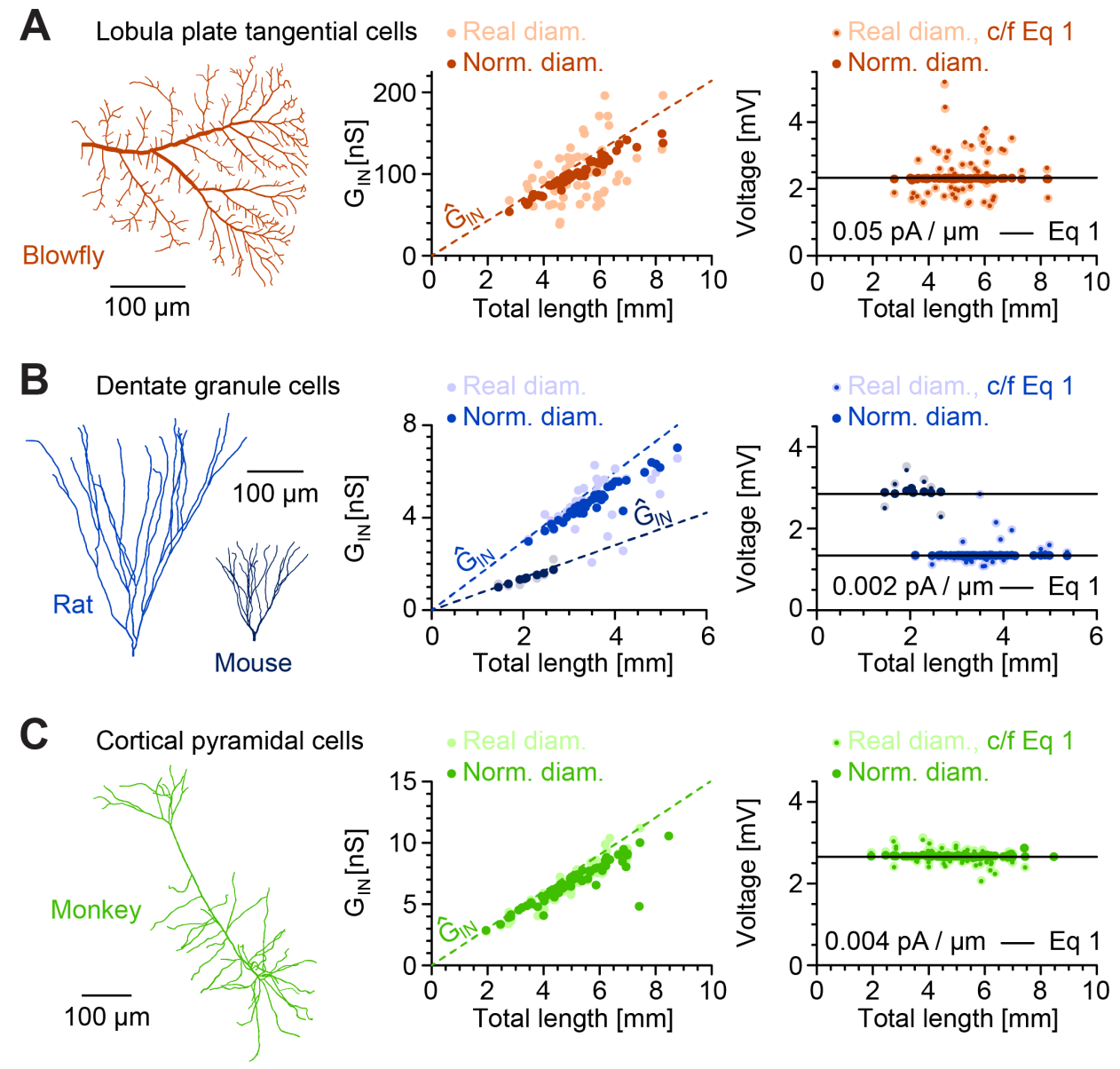

Fig 2. Passive steady-state model responses to distributed synaptic inputs are independent of dendrite length, topology and diameter distribution.

Sample morphologies (left), their input conductances (middle panels) and responses to steady-state distributed inputs (rightmost panels) compared with the prediction from Equation 1. A, Blowfly Lobula Plate tangential cell (TC) dendrites (red, $n=55$ ) with $G_{m}$ of $500 \frac{\mu \mathrm{S}}{\mathrm{cm}^{2}}$; B, Dentate gyrus granule cells (GCs) of rat (light blue, $n=43$ ) and mouse (dark blue, $n=8$ ) with $G_{m}$ of $26.3 \frac{\mu S}{c m^{2}}-$ differences in the species come from different average diameters in the two populations; C, Monkey cortical pyramidal cell (PC) dendrites (green, $n=69$ ) with $G_{m}$ of $38 \frac{\mu S}{\mathrm{~cm}^{2}}$. Each dot corresponds to one morphology; lighter dots are original morphologies without diameter normalisation. Large darker dots are results for morphologies with diameters normalised to the average of their respective population; small darker dots are individual predictions from Equation 1 for each non-normalised morphology with its respective average diameter. Straight black lines show predictions from Equation 1 using the average diameter of each population of morphologies and their respective $G_{m}$. The dashed lines show the collapsed input conductance $\hat{G}_{I N}$ as it increases linearly with the total amount of cable. 


\section{Passive responses encode percentage of active synaptic inputs in a manner ${ }_{123}$ that is largely independent of branching topology and dendrite length

Importantly, the principle of dendritic constancy found in the construction of the simple cable 125 with constant diameter can be generalised to branched and tapered neuronal morphologies. 126 We show this at the example of Lobula Plate tangential cells (TCs, $n=55$ ) in the blowfly, 127 dentate gyrus granule cells (GCs) in rat $(n=43)$ and mouse $(n=8)$ and cortical pyramidal 128 cells (PCs, $n=69$ ) in the monkey with their respective $G_{m}$ under steady-state distributed 129 inputs (Figure 2). These three datasets were chosen to represent a very leaky large cell (TC), 130 a small and electrotonically compact cell (GC) and the most typical cortical cell (PC) from a range of different species. Normalising the average diameters to the overall average diameter $d$ of the respective datasets shows that the steady-state responses are independent of branching patterns and diameter taper (compare larger dark dots with black lines in rightmost panels in Figure 2). In addition, the individual voltage responses of each cell with their original diameters were well predicted by Equation $\mathbf{1}$ (small dark dots in rightmost panels of Figure 2) with normalised root mean square errors (nRMSE) of $1.3 \%$ for TCs, $0.9 \%$ for GCs, and $1.2 \%$ for PCs.

Our prediction also accounts for responses to a smaller proportion of activated synapses, i.e. a 139 lower synaptic density, with voltage responses linearly relating with the percentage of active synapses. However, it is important to show what effect a specific, more clustered, distribution of synapses would have on the overall responses in individual neurons. We therefore titrated for any given percentage of active synapses the two most extreme distributions: We compare voltage responses to the activation of a given proportion of the most distal (Figure 3, solid lines) and, respectively, the most proximal (Figure 3, dashed lines) synapses. Even under such clustering of active synapses, neurons seemed to be able to encode the percentage of active synapses with their root voltages both in the steady-state (Figure 3, middle panels) and in dynamic simulations (Figure 3, right panels) following Equation $\mathbf{1}$ that includes the synaptic conductances present in these simulations. Importantly, passive somatic voltage responses reflected the percentage of active synaptic inputs independently of morphological complexity and dendrite length (compare Figure $3 \mathrm{~A}$ with Figures $3 \mathrm{~B}$ and $\mathrm{C}$ ). 
A Blowfly Lobula plate tangential cells
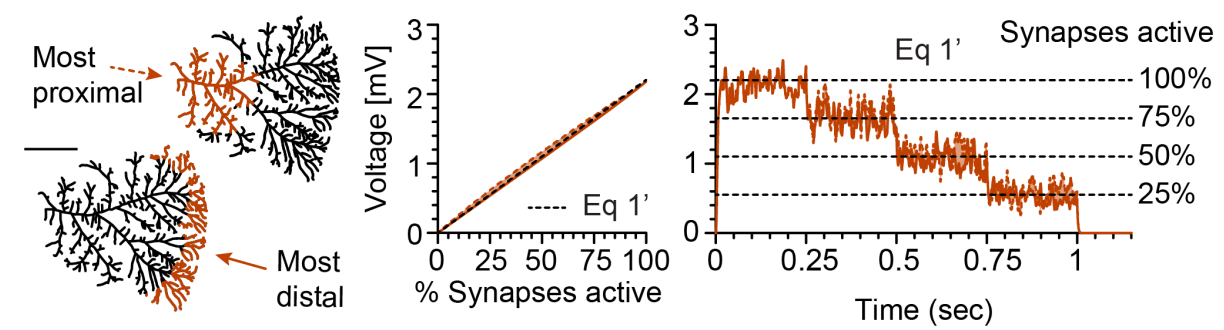

B Rat dentate granule cells
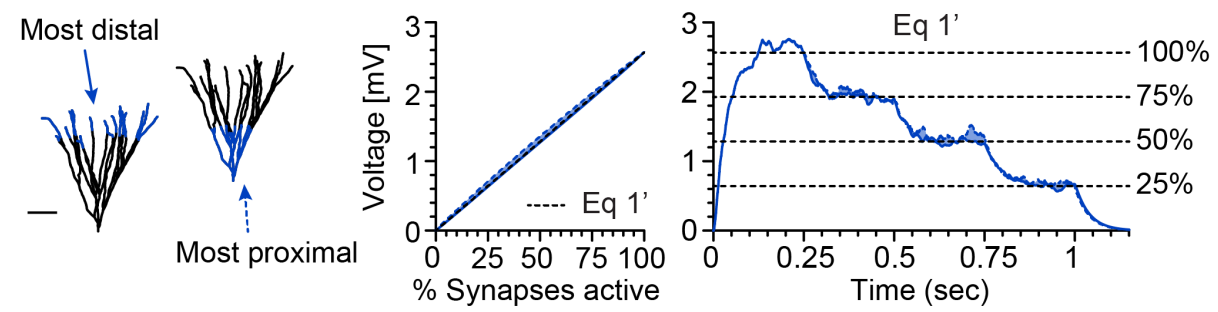

C Monkey cortical pyramidal cells
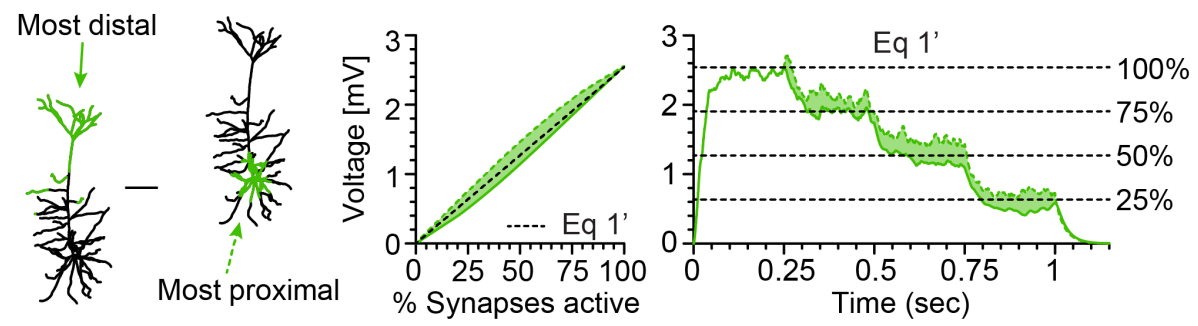

Fig 3. Passive model voltage responses follow relative percentage of active synapses even when these are clustered.

Synapse distributions (left), steady-state responses to partial activation of synapses (middle) and responses to sample levels of $(100 \%, 75 \%, 50 \%, 25 \%$ and $0 \%)$ in dynamic simulations (rightmost panels). A, B, and C, each single out one morphology (the one shown on the left) from the populations used in Figure 2 (using the same colour scheme). Dashed coloured lines are the responses to the most proximal synapses while solid coloured lines show the responses to the activation of the most distal synapses. The space in between both responses is shaded. For example, the $25 \%$ line means that the $25 \%$ most proximal synapses were active (dashed lines) and in a second simulation the $25 \%$ most distal synapses were active (solid lines). Black dashed lines are predictions from Equation $\mathbf{1}^{\prime}$ that include the synaptic conductance. Scale bars show $100 \mu \mathrm{m}$.

Next, we tested whether the principle of dendritic constancy holds across diverse dendrite 152 branching patterns and sizes in a large number of different cell types. Indeed, our calculation ${ }_{153}$ for the steady-state voltage response to distributed inputs in the simple cable yielded good 154 predictions for the wide range of real dendritic morphologies from the July 2016 version of 155 the NeuroMorpho.Org database (Ascoli, 2006). We selected those datasets (223 datasets, 9, $841{ }_{156}$ reconstructions, Table S1) that contained dendritic morphologies with sufficient detail in 157 
all three dimensions and with reconstructed diameters (see Methods). Input conductances ${ }_{158}$ and steady-state voltage responses to distributed inputs were calculated after normalising $\quad 159$ the diameters to an average $1 \mu \mathrm{m}$ and for generic values of $G_{m}$ of $50 \frac{\mu S}{\mathrm{~cm}^{2}}$ that are typical for 160 cortical pyramidal cells (Figure 44A). We observed here that very large trees exhibited a trend ${ }_{161}$ to smaller voltage responses. We found similar results in morphological models for dendritic 162 trees based on minimum spanning trees (Cuntz et al., 2007, 2010, 2012) covering a very large 163 range of possible complexities and overall sizes in synthetic dendrites (Figure S1). Also, 164 the dynamic responses to synaptic stimulation were well predicted for morphologies from 165 NeuroMorpho.Org using Equation $\left[\mathbf{1}^{\prime}\right.$ but very small trees showed strong fluctuations because ${ }_{166}$ of the small number of synapses there (Figure 4 $4 \mathbf{B}$ ). Overall, the responses were faithful to our ${ }_{167}$ prediction over a range of four orders of magnitude of dendritic length (nRMSE of $5.1 \%$ for ${ }_{168}$ the steady state responses compared with Equation 1). 

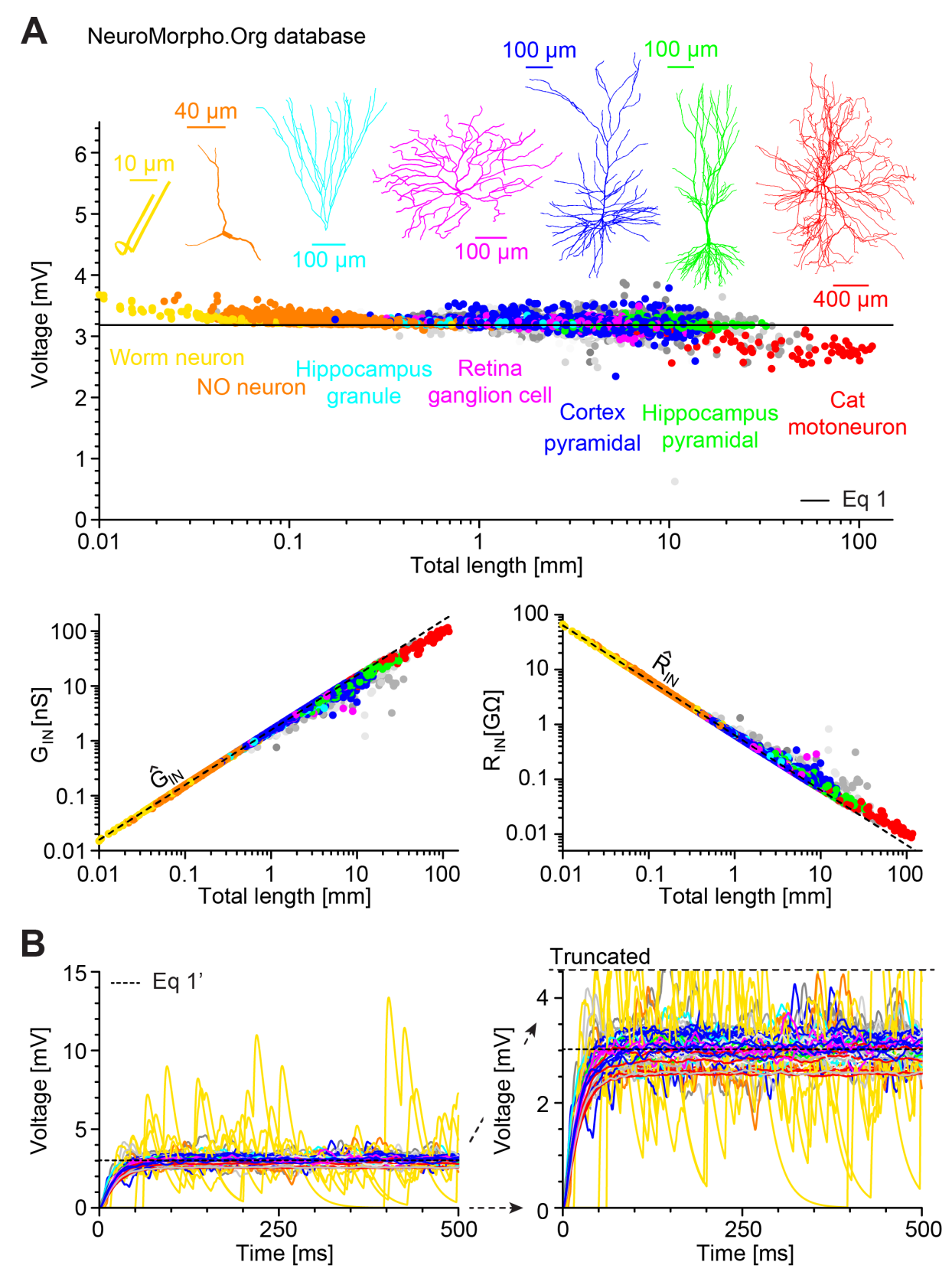

Fig 4. NeuroMorpho.Org database-wide analysis reveals neuronal size and shape invariant passive model responses to distributed inputs.

A, Voltage responses to distributed inputs with $G_{m}$ of $50 \frac{\mu S}{c m^{2}}$ in a large selection of all morphologies from NeuroMorpho.Org (223 datasets, 9, 841 reconstructions, Table S1) after normalisation of average diameters to $1 \mu \mathrm{m}$. Larger consistent subgroups are indicated by colours, representative morphology and label. Unlabelled smaller groups are different shades of grey in the background. The straight line indicates the analytical prediction from Equation 1 for an unbranched cable. Input conductances $G_{I N}$ (left bottom) and input resistances $R_{I N}$ (right bottom) are indicated in the same colour code as in the top panel and compared to the case where the overall membrane was collapsed in $\hat{G}_{I N}$ and $\hat{R}_{I N}$ respectively (dashed lines). B, Passive dynamic responses and prediction from Equation $\mathbf{1}$ (dashed line) in the first morphology of each of the 223 datasets, similarly to the three morphologies in Figure 3 , rightmost panels. Since small worm neurons (yellow) exhibited large fluctuations around the mean, this panel is shown at two different scales. 


\section{Spike frequency but not temporal sequence of spikes is independent of 170 model dendrite shape and size in response to distributed synaptic inputs

So far, we have shown a fundamental aspect of passive normalisation of the neural response to synaptic inputs that seems true for all dendritic morphologies. In the following, we used a classical spiking model of cortical pyramidal cells (Mainen et al., 1995; Mainen and Sejnowski, 1996) to test whether our principle of dendritic constancy translates to active spiking neurons. When incorporated in diverse neuronal morphologies from different cell types, this spiking mechanism was previously shown to exhibit strongly varying spiking patterns for somatic current injections (Figure 5A, top row; similar analysis to the original paper using the model \#2488 from ModelDB however with normalised average diameters for a better comparison with our predictions, see Methods) (Mainen and Sejnowski, 1996). To quantify the spiking behaviour in four different cell types we plotted firing rates as a function of injected current into the soma (f-I-curves, Figure 5B, top panel). As expected, the spiking frequency increased with decreasing dendrite size rendering smaller cells more excitable. We used interspike interval (ISI) distributions to characterise the temporal structure of the spike trains (bursting vs. non-bursting) in the different morphologies (Figure 5B, bottom panel, ISI distribution refers to single cell firing). L5 pyramidal cells exhibited bursts of three spikes (as indicated by a larger proportion of short ISIs) and L3 pyramidal cells bursts of two spikes (as indicated by two equal peaks in the ISI distributions). Interestingly, when stimulated by distributed synaptic inputs instead of somatic current injections, the differences in the temporal structure of the spiking (bursting vs. non-bursting) remained dependent on the respective morphology with similar ISI distributions as well as coefficients of variation (cv) (Figure 5C, bottom panel). However, the numbers of spikes were equalised and were independent of dendritic tree size irrespective of the frequency of stimulation (Figure $5 \mathbf{A}$, bottom traces, and Figure $5 \mathrm{C}$, top panel). The equalised passive voltage responses predicted by our dendritic constancy (Figure 5C, rightmost panel, bottom traces, subthreshold) were transformed into equal number of spikes in the active model (Figure 5C, rightmost panel, top traces). Taken together, in response to distributed synaptic inputs, spike numbers (frequencies) were independent of morphology while spike times (as reflected in the temporal structure of the spiking in the form of bursting vs. non-bursting) remained affected by morphological properties of dendrites in line with previous observations by Mainen and Sejnowski (1996) for responses to somatic current injections. 

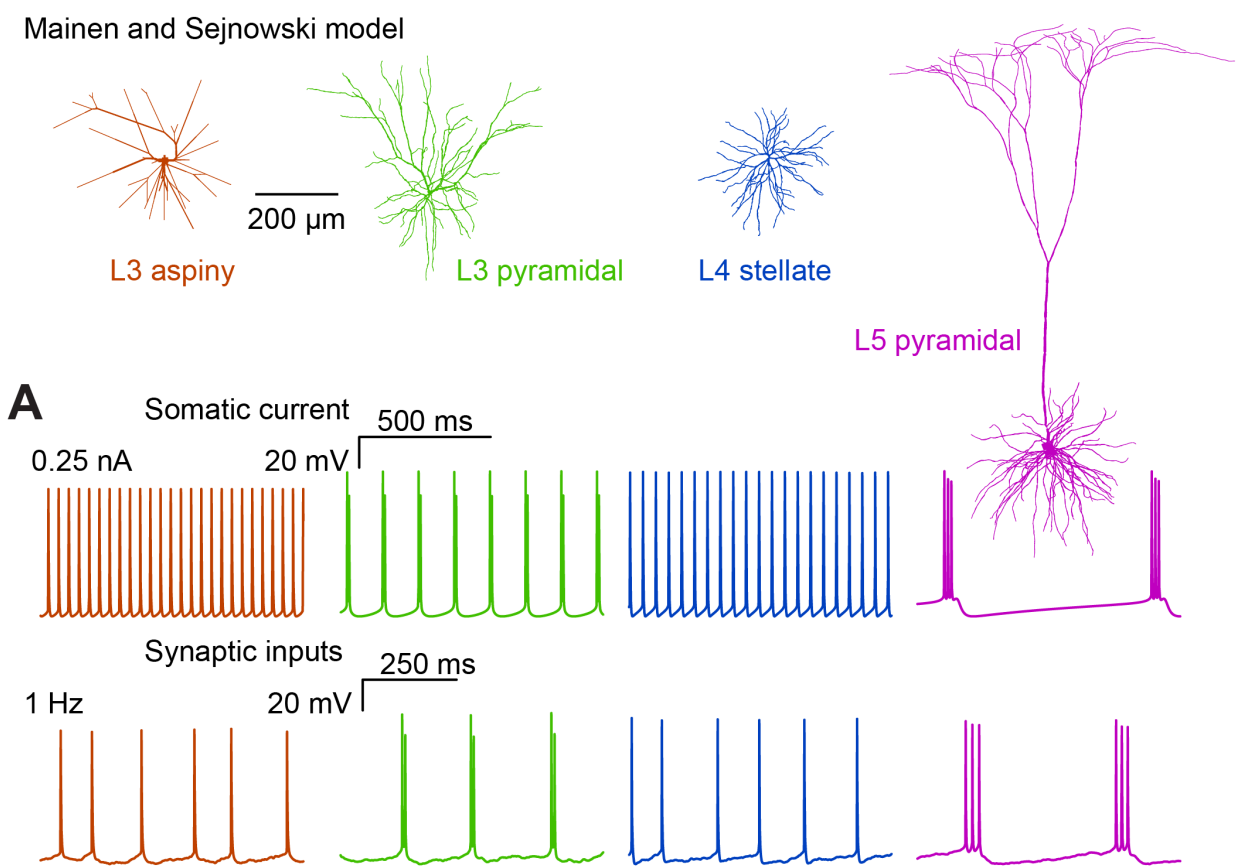

B Somatic current

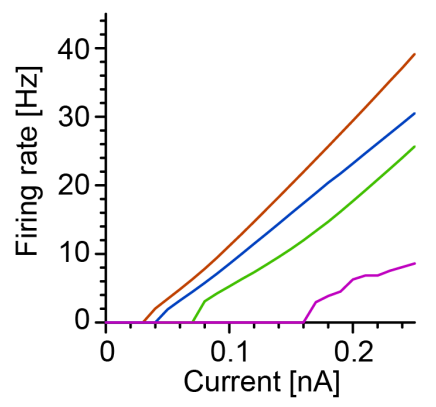

C Synaptic inputs
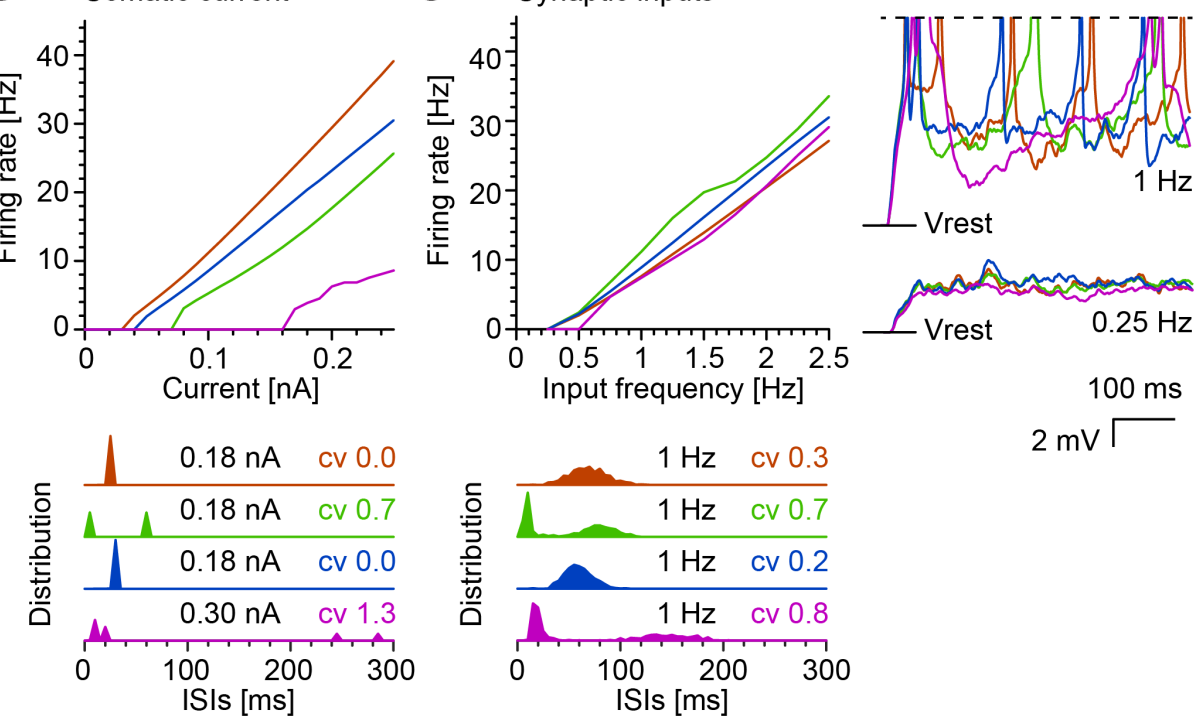

Fig 5. Neuron size- and shape-invariant responses to distributed inputs in spiking neurons.

Simulations using the spiking mechanism by Mainen and Sejnowski (1996) in their four sample morphologies of L3 aspiny (dark orange), L3 pyramidal (green), L4 stellate (dark blue), and L5 pyramidal (pink) cells after normalising the diameters. A, Sample voltage traces for $0.25 n A$ current injections into the soma (top, similar to Figure 1 in the original work; see Methods) and distributed synaptic inputs at $1 \mathrm{~Hz}$ (bottom). B, Firing rate vs. current injection in the soma and $\mathbf{C}$, responses to distributed synaptic inputs for the same four cases. Sample subthreshold (bottom, $0.25 \mathrm{~Hz}$ ) and suprathreshold (top, $1 \mathrm{~Hz}$; truncated spikes at dashed line) synaptic activation in the four morphologies are shown in the rightmost panels. Interspike interval (ISI) distributions are shown below the respective panels for $40 \mathrm{sec}$ simulations at indicated current injections and synaptic activation with corresponding coefficients of variation (cv). Note the two peaks in ISI distributions of L3 and L5 pyramidal cells indicative of their bursting. Colours indicate the different morphologies from A throughout the figure. 
In order to verify that the results in the model by Mainen and Sejnowski were not model- 202 specific, we performed similar simulations in two distinct well-established active models 203 of CA1 pyramidal cells by Jarsky et al. (2005) and Poirazi et al. (2003b). We integrated the ${ }^{204}$ corresponding active ion channel models into the set of all good reconstructions of hippocam- 205 pal pyramidal cell morphologies $(n=105)$ from NeuroMorpho.Org after normalising their 206 diameters to $1 \mu \mathrm{m}$ (Figure 6A). The model by Jarsky et al. has previously been used to study 207 the separate effects of inputs from Schaffer collaterals (SC) and the perforant path (PP) (Jarsky 208 et al., 2005). This gave us the opportunity to compare our results for distributed inputs over 209 the entire dendrite with results for inputs that were clustered in a more realistic manner 210 according to their anatomical (layer-specific) origin. In this case we compared stimulating ${ }^{211}$ all synapses with $1 \mathrm{~Hz}$ (Figure $6 \mathbf{B}$, black dots) and, separately, only synapses impinging on ${ }_{212}$ the basal dendrites (Figure $\mathbf{6 B}$, red dots) or on the distal apical dendrite and tuft region ${ }_{213}$ (Figure 6B, cyan dots). Remarkably, in all cases, the firing rates were independent of neuron ${ }_{214}$ size. Again, the number of spikes was indicative of the percentage of active synapses scanned 215 in a similar manner to Figure 3 (Figure 6 $\mathbf{B}$, rightmost panel). In particular, the corresponding 216 input-output functions were almost identical when measured in two different sample mor- ${ }_{217}$ phologies of radically different total dendritic length (Figure 6B, rightmost panel, compare ${ }_{218}$ both sets of solid and dashed lines).

A second model of CA1 pyramidal cells by Poirazi et al. (2003b) has become archetypal for 220 compartmentalised computations in dendrites. Similarly to the model by Jarsky et al., we 221 incorporated the ion channel models by Poirazi et al. into the NeuroMorpho.Org collection 222 of hippocampal pyramidal cell morphologies and subjected the individual compartmental ${ }_{223}$ models to various combinations of distributed synaptic inputs. The model by Poirazi et 224 al. produced large dendritic events that were distinct from the somatic action potentials 225 (Figure 6C, voltage traces red - dendrite vs black - somatic). Intriguingly, both numbers 226 of somatic spikes and large dendritic events were independent of total dendritic length 227 (Figure 6C, left lower and upper panels respectively). 
A CA1 pyramidal cells

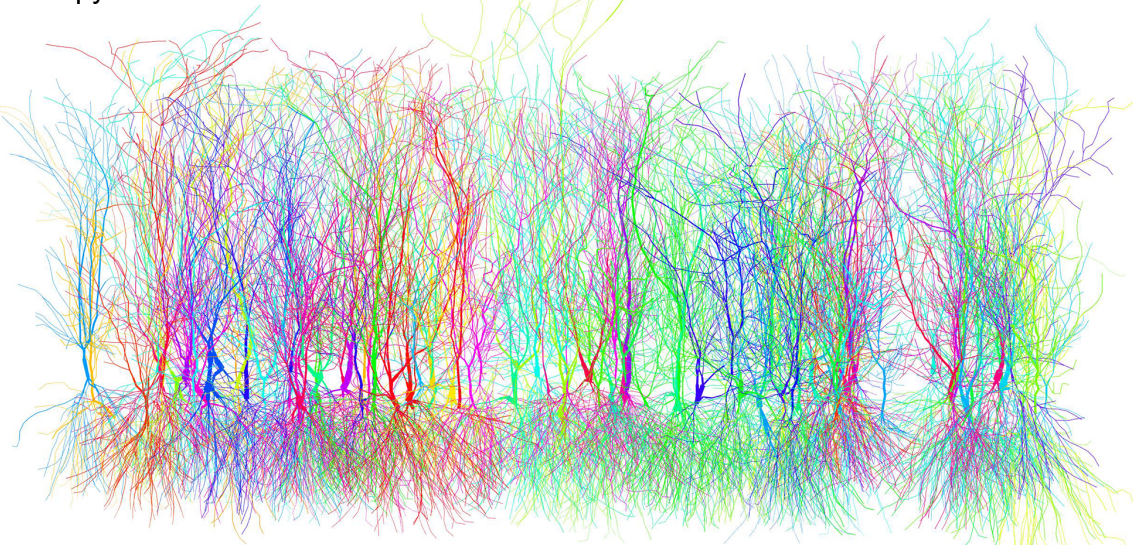

B Jarsky et al. at $1 \mathrm{~Hz}$ inputs
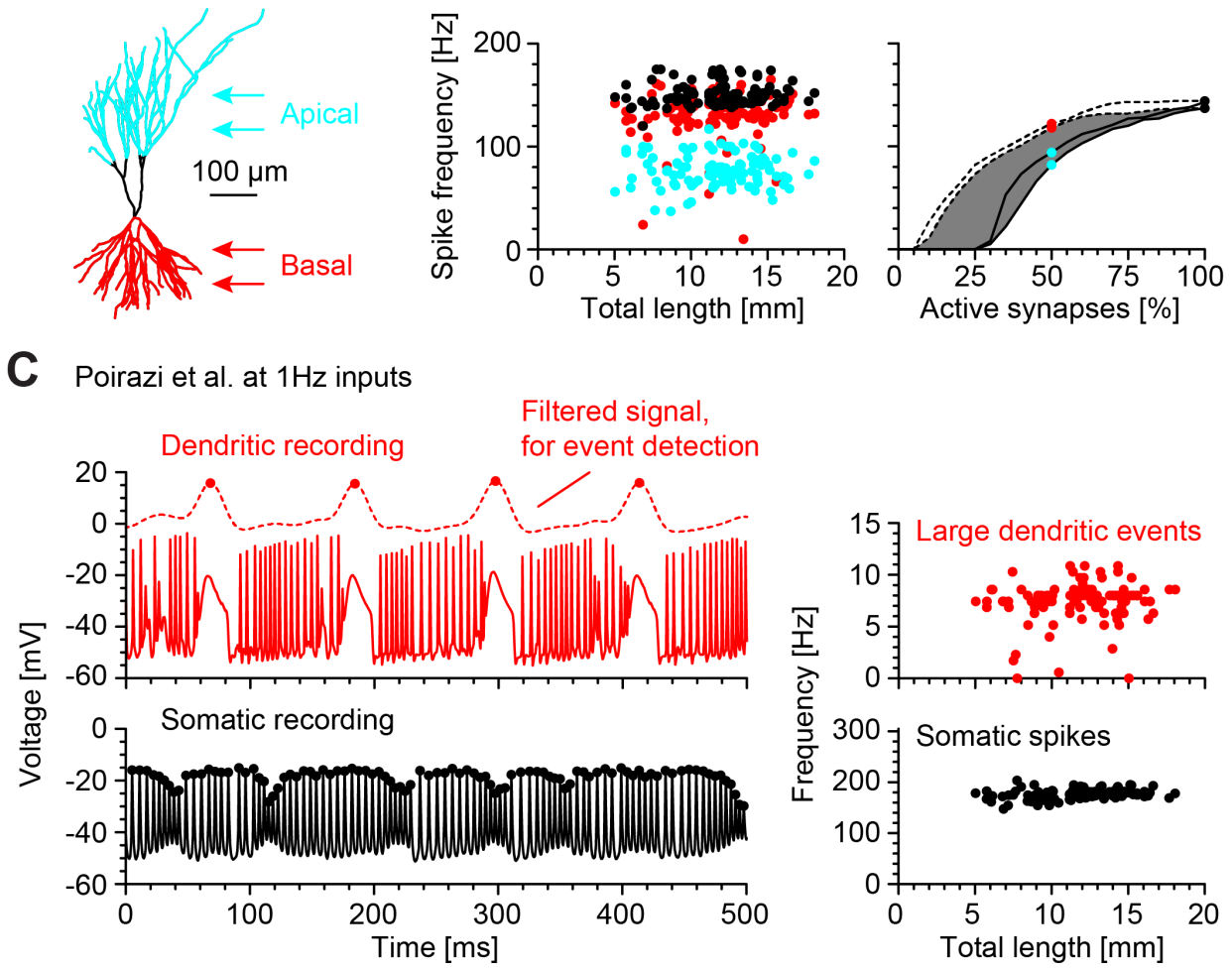

Fig 6. Dendritic constancy in two active models of hippocampal CA1 pyramidal neurons including dendritic spikes and clustered inputs.

A, All 105 morphologies of rat hippocampal pyramidal cells from NeuroMorpho.Org that passed our manual curation criteria (Colours are random). B, CA1 pyramidal cell model by Jarsky et al. (2005) with its responses to distributed synaptic inputs $500 \mathrm{pS}, 1 \mathrm{~Hz}$ in the set of all 105 morphologies. Black dots show spiking responses to activation of all synapses while red (basal) and cyan (apical) show responses to activation of subregions of the dendrite as indicated in the sketch on the left. Rightmost panel shows spike output analysis for selective activation of a subset of all most proximal (dashed line) and most distal synapses (solid line). Since roughly $50 \%$ of synapses were active in both the basal and apical stimulations, corresponding values of the curve for proximal (red) and distal (cyan) synapses are highlighted in the rightmost panel as well as values for all synapses (black). The two sets of curves (two dashed, two solid lines, respectively) represent two morphologies of radically different size (10 $\mathrm{mm}$ vs. $15 \mathrm{~mm}$ of total dendritic length). See next page. 
Fig 6. (continued) The shaded area highlights the range of responses in clustered synapses for the morphology shown in the inset. C, CA1 pyramidal cell model by Poirazi et al. (2003b) driven by distributed synaptic inputs $500 \mathrm{pS}, 1 \mathrm{~Hz}$ in the set of all selected 105 hippocampal pyramidal cell morphologies from NeuroMorpho.Org. Top row, Large dendritic events (red dots) were measured at the branch with highest $y$ value respectively and detected after low pass filtering the local voltage signal there with a Gaussian filter with variance of $100 \mathrm{~ms}$ (see dashed line). Bottom row, Somatic events (black dots) as measured directly from the somatic voltage, shown for one sample morphology. Rightmost panels show resulting frequency of somatic (black, bottom) and dendritic (red, top) events with one point each per morphology as a function of total dendritic length.

\section{Spiking reset converts constant membrane voltage into constant spike rates}

The results from the active compartmental models pose the question as to why the con- 230 stant voltages transform into constant numbers of spikes. Such a transformation could be a consequence of each spike essentially shunting and resetting the entire neuron (Häusser, 2001) while erasing its voltage history. Under these assumptions, a leaky integrate-and-fire (LIF) (Stein, 1965) mechanism coupled to the dendrite could help elucidate the constancy of spike numbers. We chose to implement a LIF that resets the voltage throughout the entire dendritic tree after passing a threshold voltage at the dendrite's root. Incorporating such a spiking mechanism in the four cell types of Figure 2, yielded an output spiking frequency that was indeed independent of dendritic length (Figure 7A) for any given synaptic input frequency. In fact, the entire input-output $(\mathrm{IO})$ curves were essentially independent of the morphology (Figure 7B). We then derived an analytical solution for the transformation of variable synaptic input activity into firing rate output (see Methods, Equations 10-22). In line with our numerical LIF simulation results (Figure 7 A, and B), the mean analytical voltage response to stochastic inputs in a uniform cable was independent of length. The variance of the subthreshold voltage response decayed to a constant for dendrites of total electrotonic length greater than one. Our analytical predictions for the IO relationship (Brunel and Hakim. 1999) are

$$
R^{-1}=\tau \int_{0}^{\infty} \frac{1}{z} e^{\frac{-z^{2}}{2}}\left(e^{z \frac{v_{t h}-\mu_{v}}{\sigma_{v}}}-e^{z \frac{v_{r e}-\mu_{v}}{\sigma_{v}}}\right) d z
$$

with firing rates $R$ for subthreshold voltage mean $\mu_{v}$ and standard deviation $\sigma_{v}$ impinging ${ }^{247}$ on a membrane with time constant $\tau$, firing threshold $v_{t h}$, and voltage reset $v_{r e} . R$ always ${ }^{248}$ converges to constant values for cable lengths longer than the electronic length (see Methods, 249 Equations 10-22, Figure 7B bold black lines). Importantly, $R$ is practically independent of 250 dendritic length, if the mean afferent drive is sufficiently strong and so the output firing rate 251 
is less dependent on fluctuations (as seen in Figure 7 $\mathbf{A}$ ). In our case in Figure $7 \mathbf{B}$, the specific ${ }_{252}$ membrane conductance in the different cell types determined the slope of the IO curves with ${ }_{253}$ a very sharp slope in the leaky blowfly TC that in reality produces spikelets and shallower ${ }^{254}$ curves in the other cell types with lower conductivity through the membrane. Interestingly, 255 the percentage of active synapses was encoded in the number of spikes (Figure 7C) in analogy ${ }_{256}$ to the voltages in Figure 3 (compare with IO curves in Figure 7B). Again, this was true even ${ }^{257}$ for clustered synapses (plots show most distal synapses as solid lines vs. most proximal 258 synapses as dashed lines). 
Leaky integrate-and-fire (LIF) models

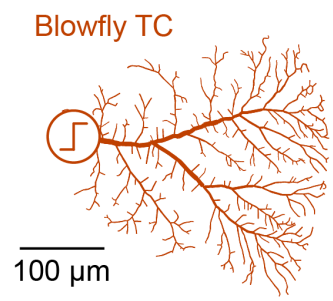

A
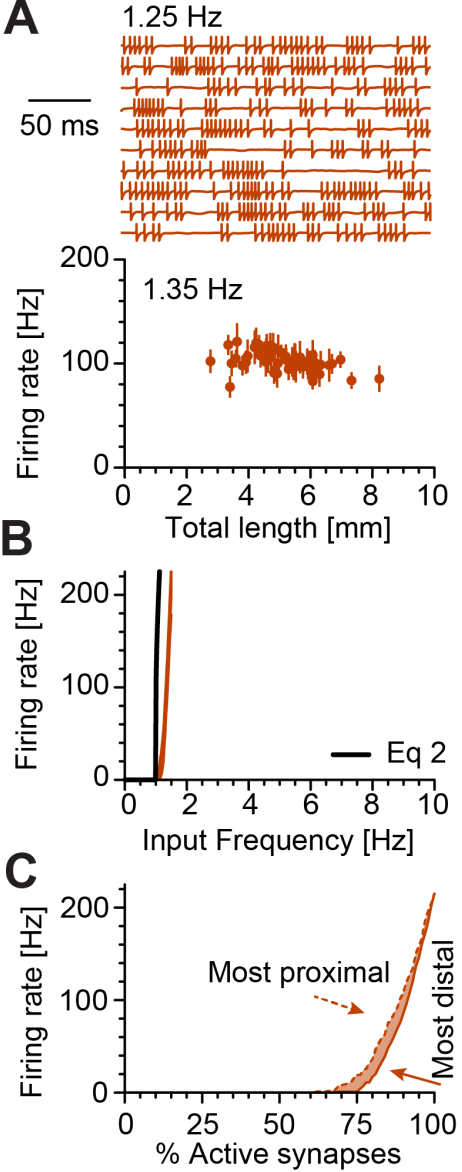
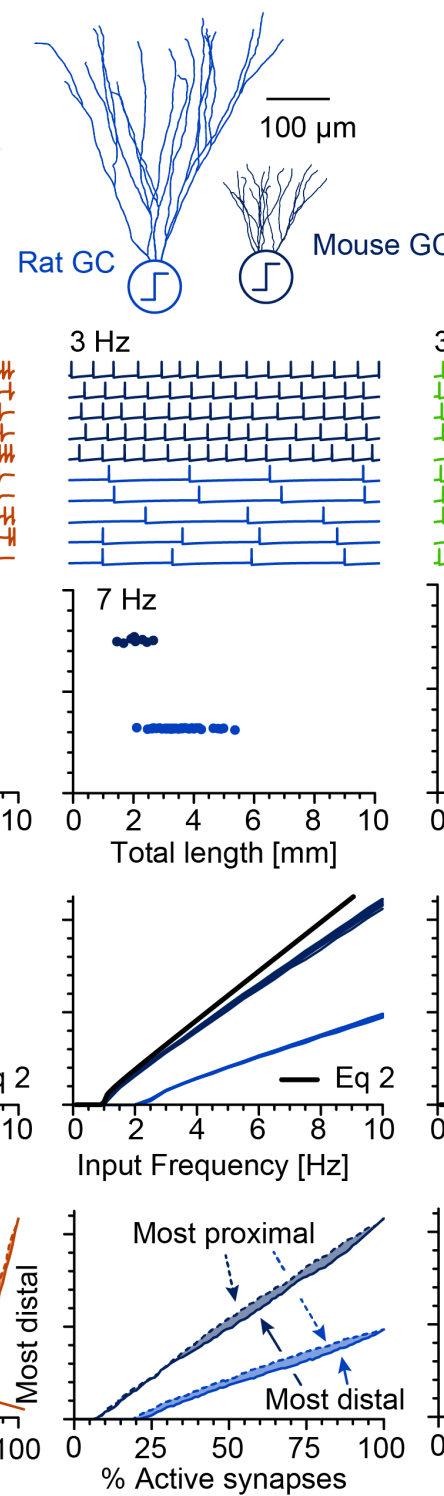
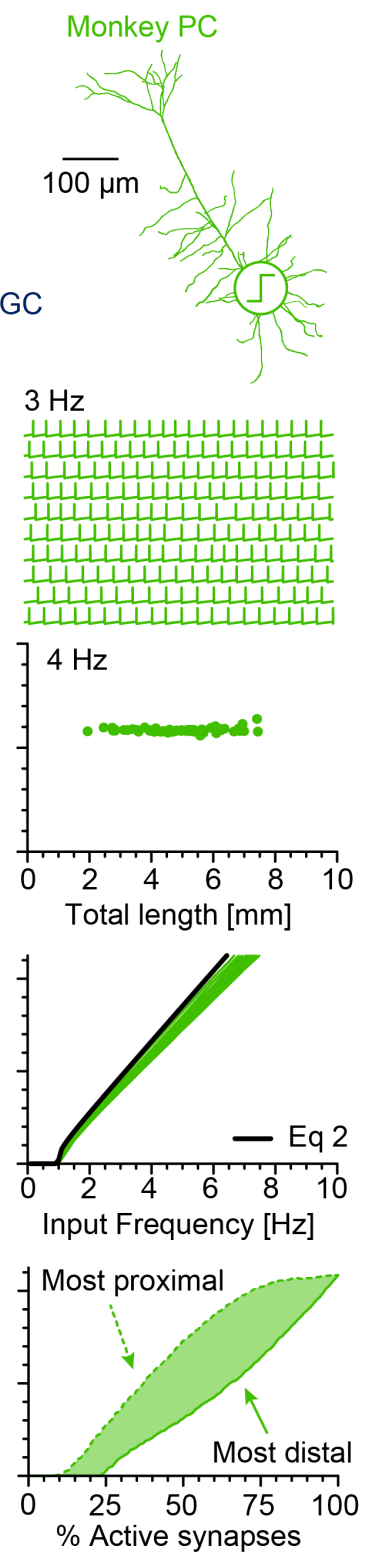

Fig 7. Spiking responses to distributed inputs in leaky integrate-and-fire (LIF) neurons with realistic dendritic morphologies as the source of leak indicate that voltage reset throughout the entire dendritic tree contributes to spike rate constancy.

LIF mechanism in its simplest form (without adding soma or axon, the LIF resets the voltage in the entire dendrite after spiking) introduced into the dendritic root of the cell types from Figure 2 with the same colours and membrane properties. A, Top row, Sample spike trains for 10 different TC, 5 rat GC, 5 mouse GC and 10 monkey PC dendritic morphologies are shown underneath the corresponding morphologies. Bottom row, Firing rates are shown with error bars (standard deviation, invisible in GCs and PCs) for one selected input frequency for each cell type are shown as a function of length. B, Input-Output (IO, Frequency of synaptic activation vs. spiking frequency) plots for all available morphologies for TCs (left), mouse and rat GCs (middle) and monkey PCs (right). Respective cable calculations from Equation 2 that are independent of length are shown as bold black lines. C, Responses to selective activation of the most proximal (dashed lines) and most distal (solid lines) synapses in the dendrite. The areas between these two extreme scenarios for clustered synapses are shaded. 
Similarly, incorporating the LIF mechanism into the dendritic morphologies of the Neuro- ${ }_{260}$ Morpho.Org database (with a uniform specific membrane conductance, see Figure 4) yielded 261 invariant IO curves over a very large range of morphologies. Only the IO curves from tiny 262 worm neurons (yellow) and very large spinal cord motoneurons (red) deviated from the re- 263 maining curves (Figure 8 A, LIF model, compare also these results with analytical predictions ${ }_{264}$ from Equation 2). In the same morphologies, we also showed spike number invariance using 265 an adaptive exponential LIF (AdExpLIF) (Brette and Gerstner, 2005) for two specific temporal 266 patterns of spikes typically seen in compartmental models, a bursting mode and spiking mode ${ }_{267}$ with spike frequency adaptation (Figure $8 \mathrm{~B}$ and C, AdExpLIF model). Overall, LIF based ${ }_{268}$ spiking models were consistent with the dendritic constancy of passive voltage responses to 269 distributed synaptic inputs transforming into constant spike numbers that were independent 270 of dendritic length or shape. 
Leaky integrate and fire (LIF) in NeuroMorpho.Org dendrites
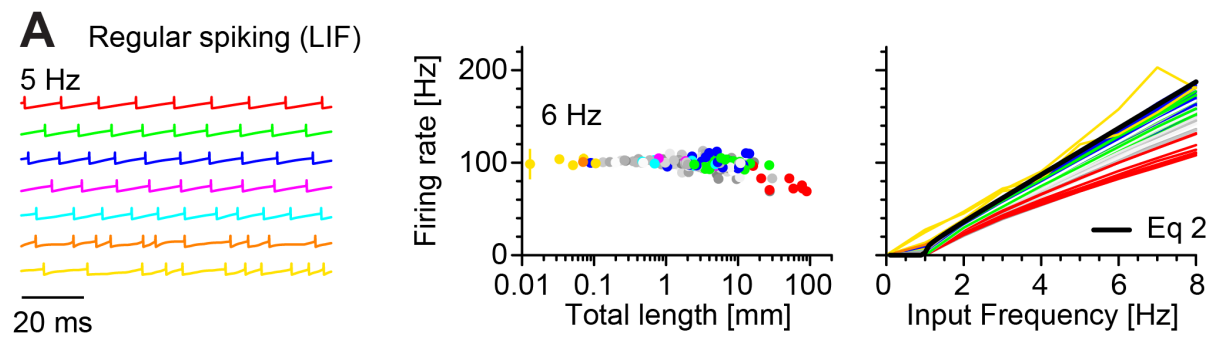

Adaptive exponential LIF (AdExpLIF) in NeuroMorpho.Org dendrites
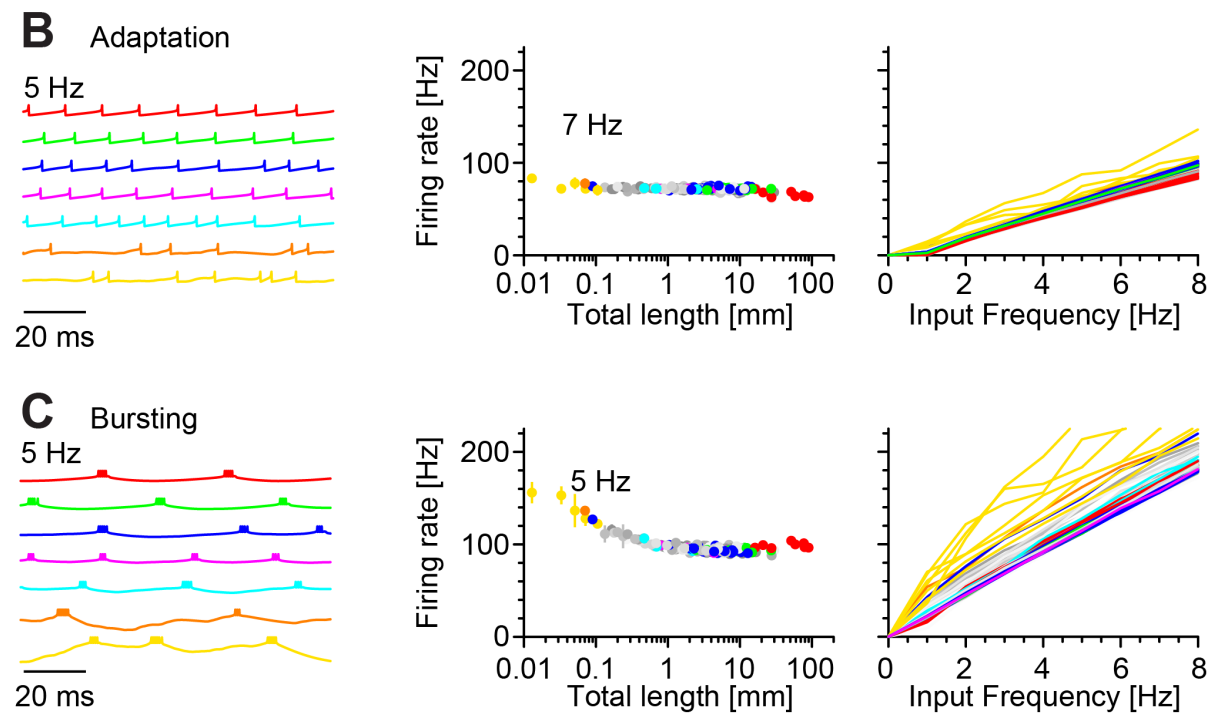

Fig 8. NeuroMorpho.Org database-wide spiking responses to distributed inputs in leaky integrateand-fire (LIF) and adaptive exponential LIF (AdExpLIF) neurons are independent of dendritic size and shape.

Similar panels as in Figure 7A and B but for all morphologies from Figure 4 with the respective membrane properties used there: Sample voltage traces in sample morphologies for all seven morphological categories (left panels), firing rates in response to a given input frequency (middle panels, input frequency is indicated), and IO curves (right panels). Colours as in Figure 4 in increasing dendrite size: Yellow (worm neurons); orange (nitergic neurons); cyan (hippocampal granule cells); pink (retinal ganglion cells); blue (cortical pyramidal cells); green (hippocampal pyramidal cells); red (cat motoneurons). A, Regular leaky integrate-and-fire (LIF) spiking mechanism incorporated in all dendritic morphologies. Similarly to Figure 7 . calculations from Equation 2 are shown as a bold black line. B, Adaptive exponential LIF (AdExpLIF) that includes an additional channel for spike frequency adaptation and results in less regular spiking. C, Long time constant in the adaptation channel of the AdExpLIF for testing our dendritic constancy theory under extreme burst firing. 


\section{Discussion}

In this work, we used analytical methods to demonstrate a general principle of dendritic constancy regarding the voltage and spiking responses to distributed synaptic inputs. Synaptic inputs effectively encounter an apparent input conductance in the soma (i.e. the transfer conductance) corresponding to the collapsed membrane leak of the entire dendrite onto the soma. As a consequence, more synaptic currents in larger cells are precisely compensated by the additional dendritic leak. Our dendritic version of Ohm's law (Equation 1 and Equation $\mathbf{1}^{\prime}$ as well as Equation 2 for spikes with variable inputs) is independent of morphological features and spiking mechanisms and predicts isoelectrotonic behaviour for anatomically distinct dendritic trees shaped by species-specific scaling (Beining et al., 2017; Cuntz et al., 2013), developmental expansion (Mckay and Turner, 2005) or neurodegenerative shrinkage (Platschek et al., 2016). Finally, our simulations in a classical model by Mainen and Sejnowski (1996), as well as other established spiking models (Jarsky et al., 2005; Poirazi et al., 2003b) and LIF models showed that synaptic stimulation in different dendritic trees leads to similar responses in terms of firing rates but not patterns (spike times). This was true for uniformly activated synapses but also to a large degree for clustered synapses, so much so, that the somatic firing rate allowed for an approximate estimation of the percentage of active synapses independent of their dendritic location. Taken together, our analytical and numerical results imply that the principle of voltage and spike rate constancy is general since it holds in all (branched and unbranched) dendritic arbours activated by distributed synaptic conductances.

\section{Limitations for dendritic constancy}

What are the assumptions and limitations of our computational analysis? First, dendritic constancy will be affected by a number of dendritic features. The voltage responses do depend on the specific membrane conductances and average dendritic diameters. While average dendritic diameters do not seem to vary much in the NeuroMorpho.Org database (Figure S2), $G_{m}$ values are known to vary between cell types (Borst and Haag, 1996) (Figures 2, 3 and 7) and also within cell types (e.g. Garden et al., 2008). In addition, the somatic membrane leak affects the dendritic constancy results when somata are very large compared to the overall dendritic membrane if their diameters are not normalised together with the dendrites (i.e. if they do not scale with dendrite length) and if they do not receive synaptic inputs (Figures $\mathbf{S 2}$ and S3). 
Second, in our analysis, we assumed uniform kinetics, conductances and reversal potentials of synaptic inputs. In this respect, it would be interesting to further explore models with variations in distance-dependent synaptic properties (Häusser, 2001: Magee and Cook, 2000) as well as democratising effects on distal synapses (London and Segev, 2001; Rudolph and Destexhe, 2003). Here, we show for a distance-dependent linear gradient of maximal synaptic conductance (increasing with dendritic path length from the root) that dendritic constancy is preserved in the hippocampal CA1 pyramidal cell model by Jarsky et al. and in the NeuroMorpho.Org morphologies (Figures S4A and B). Furthermore, our analytical solutions predict that dendritic constancy applies also to inhibitory synapses with negative reversal potentials (same models, Figures S5A and B). However, the effects of layer-specific somatic or dendritic inhibition, introducing local or distant shunts (Gidon and Segev, 2012) and their interaction with active channels (see below) need to be studied in detail.

Third, it also remains unclear how dendritic non-linearities such as dendritic NMDA or 315 calcium spikes would operate in the context of our dendritic constancy principle. Such ${ }_{316}$ non-linear computations would include dendritic integration features known to play a role ${ }_{317}$ depending on relative locations of synaptic inputs (Branco et al., 2010; Cuntz et al., 2003; 318 Poirazi et al. 2003b; Polsky et al., 2004). Surprisingly, our active cortical and hippocampal ${ }_{319}$ models exhibited dendritic constancy (without requiring any specific tuning) of spike numbers ${ }_{320}$ and even in the numbers of dendritic active events in the model by Poirazi et al. (Figures 6C). ${ }_{321}$ However, many further aspects related to dendritic voltage-dependent channels remain to 322 be explored. For instance, potassium channels and H-channels are capable of affecting local ${ }_{323}$ synaptic potentials as well as backpropagating spikes (Chen et al., 2006; Magee, 1999). It ${ }_{224}$ would be intriguing to test how these channels shape dendritic constancy for synchronous or 325 asynchronous, clustered or distributed synaptic inputs.

Fourth, it remains to be determined how dendritic constancy might interact with recently 327 described homeostatic plasticity of the axon initial segment (AIS) in the form of activity- ${ }_{228}$ dependent changes in its location and length and in the distribution of its ion channels (Adachi ${ }_{329}$ et al. 2015; Kuba, 2012). Decreased or increased synaptic activity can induce homeostatic 330 lengthening or shortening of the AIS with a compensatory increase or decrease in neuronal ${ }_{331}$ excitability respectively (Evans et al., 2015; Kuba et al., 2010). However, the effect of AIS ${ }_{332}$ length or location on excitability is more complex and depends on neuronal size (Gulledge ${ }_{33}$ and Bravo, 2016). Therefore, further computational and experimental analyses are needed to ${ }_{334}$ 
better understand the link between the neuron's size and shape invariant excitability that we ${ }_{335}$ describe here and AIS plasticity.

Of particular interest is our observation that spike times rather than spike numbers remained affected by morphological properties of dendrites in a similar manner to responses to somatic current injections (Mainen and Sejnowski, 1996). Our simulations revealed that in the case of synaptic stimulation, both dendritic constancy as well as variability in firing patterns were maintained at the same time in active models of four different reconstructed cortical cell types. Although two cortical cell models displayed regular firing and the other two bursting, all of them generated similar firing rates. The somatic bursting behaviour has been previously explained as a consequence of delayed dendritic depolarisations and subsequent return currents from dendrites, arising due to two key factors: (1) moderate coupling resistance between somatic and dendritic regions in combination with (2) separated distributions of fast and slow active channels in soma and dendrites (Mainen and Sejnowski, 1996). Our synaptically driven simulations confirmed and extended these analyses by showing that the electrotonic mechanisms of dendritic constancy are able to normalise spike numbers in cells with different dendritic sizes and shapes without disrupting the active burst generating mechanisms. It is tempting to speculate that dendritic constancy could support homogeneous spike-rate coding across different morphologies while at the same time allowing for celltype specific spike-time coding. In other words, dendritic constancy may facilitate neuronal computations by maintaining stable firing rates while keeping variability of spike patterns (Denève and Machens, 2016; Denève et al., 2017; Gjorgjieva et al., 2016).

\section{Clinical relevance of dendritic constancy}

The dendritic constancy principle could be of clinical relevance. Changes in dendritic size and shape are hallmarks of many neurological disorders, including chronic stress (Conrad et al., 2017), stroke (Brown et al., 2010; Qin et al. 2014) and neurodegeneration (Šišková et al., 2014, Spires and Hyman, 2004). Whereas dendritic atrophy caused by direct damage to a neuron is considered part of the disease process (Šišková et al., 2014), dendritic remodelling occurring in disconnected brain areas, i.e. network damage, is most likely homeostatic and restorative in nature. For example, the perforant pathway to the dentate gyrus degenerates in aged humans and in Alzheimer's disease (Leal and Yassa, 2013; Yassa et al., 2010). As a consequence, 364 the target neurons of this pathway - dentate granule cells - retract their dendrites. This 365 
dendritic retraction is caused by denervation and not by the disease itself (Einstein et al., 366 1994). Experimental animal data have shown that such denervated and retracted granule cells 367 (Vuksic et al. 2011) eventually achieve synapse densities on their dendrites comparable to pre-denervation levels (Steward et al., 1988). In that case, the input conductance as well as the number of synapses with additional unit length would likely cancel each other out. The dendritic tree has fine-tuned itself to achieve firing rate homeostasis (Platschek et al., 2016, 2017). We show here that this feature is not specific to dentate granule cells and that synaptic excitability of neurons is size-invariant for all dendritic trees due to a general electrotonic principle. Thus, transneuronal dendritic remodelling appears to play a homeostatic role in maintaining information throughput in a partially damaged network.

\section{Practical consequences for computational modelling and input-output com- ${ }_{376}$ putation of neurons

Equations 1 and $\mathbf{1}^{\prime}$ allow for quantitative predictions of voltage responses to distributed synaptic inputs. This can be helpful for tuning large-scale morphologically realistic compartmental models (e.g. Markram et al., 2015) because by setting synaptic conductances to a specific value, it is possible to achieve a target voltage (and corresponding spike numbers from Equation 2). Thus, dendritic constancy simplifies the estimation of a neuron's behaviour within a network. For instance, for a given synaptic conductance, the frequency of synaptic activation required to reach a particular membrane voltage can be computed. From the perspective of network computations, the principle of dendritic constancy can be viewed as a mechanism for preserving stable neuronal activity in the circuit (as done in Figure 7). Intuitively, adding new synapses to a spiking network model would create more spikes. Even one additional spike can dramatically alter network dynamics (London et al. 2010). However, dendritic constancy is one possible mechanism to prevent this from happening, because the cell's number of output spikes depends on the relative number of active synapses and not on their absolute number. This means that increasing the number of synapses while adjusting the morphology accordingly would effectively not change the total number of spikes in the network.

In summary, our principle of dendritic constancy serves as an equalising homeostatic mech- 394 anism on which dendritic non-linearities and synaptic plasticity can operate (London and 395 Häusser, 2005: Turrigiano, 2017). It creates a passive backbone for the conservation of excitabil- 396 
ity converting a neuron to a reliable size- and synapse number-independent "summing point" 397 within the network (Segev and London, 2000) but at the same time, it allows for more complex 398 computations with active dendrites (Schmidt-Hieber and Nolan, 2017). Because dendritic con- 399 stancy is based on basic electrotonic properties, it applies to all neurons receiving distributed 400 excitatory or inhibitory inputs. This simple and universal principle has previously been over- 401 looked because most studies focused on neuronal firing activated by somatic current injections 402 or by few synaptic inputs instead of distributed synaptic stimulation. Dendritic constancy 403 becomes apparent after leaving the "somatocentric" and embracing the "synaptocentric" view 404 of a neuron's input-output transformation.

\section{Acknowledgments}

We would like to thank S. Jagannath, S. Platschek and S. Rozada for performing preliminary ${ }_{407}$ analyses and A. Castro, F. Effenberger and M. Schölvinck for useful discussions and comments 408 on the manuscript. The work was supported by BMBF (01GQ1406 - Bernstein Award 2013409 to H.C.; OGEAM 031L0109B to T.D.), Deutsche Forschungsgemeinschaft (CRC 1080 to T.D.), 410 University Medical Center Giessen and Marburg (UKGM; to P.J.), LOEWE CePTER - Center ${ }_{411}$ for Personalized Translational Epilepsy Research (to P.J. and T.D.) and F.Z.H. was supported 412 by the International Max Planck Research School (IMPRS) for Neural Circuits in Frankfurt. ${ }_{413}$ The authors declare to have no competing financial interests.

\section{Author contributions}

H.C., A.D.B, M.B, M.S., L.M., F.Z.H., T.D. and P.J. conceived the study and wrote the paper. 416 H.C., M.B., M.S., L.M. and F.Z.H. performed the numerical simulations and A.D.B. performed ${ }_{417}$ the analytical calculations.

\section{Materials and methods}

All passive electrotonic and leaky integrate-and-fire (LIF) simulations were done in Matlab ${ }_{421}$ (Mathworks Inc, 2015b, 2017b and 2018b) using our own open-source software package, ${ }^{422}$ 
the TREES toolbox (Cuntz et al., 2010) (www.treestoolbox. org, Interim version). TREES ${ }_{423}$ toolbox functions are marked in italic and end with a _tree suffix throughout the Methods ${ }_{424}$ section. Active compartmental model simulations were done in NEURON (Carnevale and ${ }_{425}$ Hines, 2004) using our new software T2N to communicate with the TREES toolbox in Matlab ${ }_{426}$ (Beining et al., 2017). All results were further analysed in Matlab. All dendritic morphologies ${ }_{427}$ were downloaded from www. NeuroMorpho. Org (Ascoli, 2006) in July 2016. The active model 428 for the spiking mechanism by Mainen and Sejnowski (1996) for Figure 5 used model \#2488 429 from ModelDB (Hines et al. 2004). The LIF and adaptive exponential leaky integrate-and-fire 430 (AdExpLIF) models (Brette and Gerstner, 2005) using realistic dendritic leak in Figures 7 and 8 431 were implemented in Matlab. All new functions (cgin_tree, LIF_tree, LIF_FR_tree, 432 AdExpLIF_tree) will be made available as part of the TREES toolbox on publication at WWW, 433 treestoolbox.org via Github. The code and data for all figures will be made available at ${ }_{434}$ https://zenodo.org//on publication. The code was tested on various operating systems. 435 Individual methods are detailed in the following but can best be appreciated in the actual 436 Matlab scripts.

\section{Cable equation for responses to distributed inputs.}

The voltage response at distance $x$ along a closed cable of length $l$ due to current of magnitude $I_{a p p}$ injected at the root (Rall, 1959, 1962) is

$$
v(x)=v_{0}\left[\frac{\cosh \left(\frac{l-x}{\lambda}\right)}{\cosh \left(\frac{l}{\lambda}\right)}\right],
$$

where $\lambda$ is the electrotonic length constant and $v_{0}$ is the voltage at the root:

$$
v_{0}=\frac{\operatorname{coth}\left(\frac{l}{\lambda}\right)}{G_{\infty}} I_{a p p}
$$

As transfer resistance is symmetric in dendrites (Koch and Segev, 1999; Rall et al., 1967; Rushton, 1937) this also gives the voltage $v_{x}(0)$ at the root due to current injection at a distance $x$

$$
v_{x}(0)=\frac{I_{a p p}}{G_{\infty}}\left[\frac{\cosh \left(\frac{l-x}{\lambda}\right)}{\sinh \left(\frac{l}{\lambda}\right)}\right] .
$$

To find the total voltage $V$ for currents injected along the entire cylinder, we require the ${ }_{445}$ 
integral over all synaptic sites

$$
\begin{gathered}
V=\frac{I_{a p p}}{G_{\infty} \sinh \left(\frac{l}{\lambda}\right)} \int_{0}^{l} \cosh \left(\frac{l-x}{\lambda}\right) d x . \\
V=\frac{\lambda I_{a p p}}{G_{\infty}}=\frac{I_{a p p}}{G_{m} \pi d}
\end{gathered}
$$

\section{Morphologies for passive electrotonic simulations.}

Simple cables (12.5 $\mu \mathrm{m}-12.5 \mathrm{~mm}$ length in $12.5 \mu \mathrm{m}$ steps) of constant $1 \mu \mathrm{m}$ diameter (Figures $\mathbf{1} \mathbf{B}-{ }_{448}$ D) or various dendritic morphologies (Figures $2-4$ ) were resampled to constant $1 \mu m$ internode ${ }_{449}$ resolution (using resample_tree). Individual datasets used in combination with specific 450 membrane properties were from blowfly Lobula Plate tangential cells (TCs, $n=55)\left(\right.$ Cuntz $_{451}$ et al., 2008) (Figures $2 \mathbf{A}$ and $\left.3 \mathbf{A}, G_{m}=500 \frac{\mu S}{c^{2}}\right)$, rat (Rihn and Claiborne, 1990) $(n=43){ }_{452}$ and mouse (Schmidt-Hieber et al., 2007) $(n=8)$ dentate gyrus granule cells (GCs, Fig- ${ }^{453}$ ures $2 \mathbf{B}$ and $\left.3 \mathbf{B}, G_{m}=26.3 \frac{\mu S}{\mathrm{~cm}^{2}}\right)$ and monkey cortical pyramidal cells (Luebke et al., 2015; 454 Coskren et al. 2015) (PCs, $n=69$, Figures $2 \mathrm{C}$ and $3 \mathrm{C}, G_{m}=38 \frac{\mu \mathrm{S}}{\mathrm{cm}^{2}}$ ). The three datasets were ${ }_{455}$ also used in the context of leaky integrate-and-fire (LIF) spiking models in Figures 7 . Dendrite ${ }_{456}$ morphologies from the entire NeuroMorpho.Org database were used for Figures 4 and $8 \quad 457$ by manual curation of all existing archives to select those with sufficient diameter profiles, 458 sufficient depth information in $z$, sufficiently high-quality reconstructions and no sudden 459 jumps in $z$ (selection, 223 datasets, 9, 841 reconstructions, see Table S1). The selected archives 460 were sorted by cell types into the following categories in decreasing order of total cable ${ }_{461}$ length: Spinal cord motoneurons (red), hippocampal pyramidal cells (green), neocortical 462 pyramidal cells (blue), retinal ganglion cells (pink), hippocampal granule cells (cyan), nitrergic ${ }_{463}$ neurons (orange), C. elegans neurons (yellow), and other (different shades of grey per dataset). ${ }_{464}$ These categories were chosen as representatives for the possible scales of dendrites rather ${ }_{465}$ than because they corresponded to consistent cell types. All dendrite morphologies were ${ }_{466}$ normalised to a given average diameter (to the average diameters in their specific archives for ${ }_{467}$ Figures 2 and 7 and to $1 \mu \mathrm{m}$ for Figures 4 and 8 .

\section{Passive steady-state measures for dendritic morphologies.}

The collapsed input conductance was measured by summing up the leak conductance over 470 the entire membrane surface of the dendrite using the function surf_tree. The resulting ${ }_{471}$ 
calculation is made available in the new TREES toolbox function cgin_tree. Remaining electrotonic features are all readily available from the electrotonic signature (sse_tree) as introduced previously (Cuntz et al. 2010). Briefly, all membrane and axial conductances are arranged according to the tree's adjacency matrix and the current transfer between all nodes is obtained by taking the inverse of the resulting conductance matrix. Local input resistances are then found on the diagonal of this electrotonic signature since current there is injected in the same node as the voltage is measured. Voltage responses to distributed inputs are simply the sum over the column or row of the electrotonic signature since the matrix is symmetric and the system is linear. While this method simulates steady-state distributed current injections, synaptic conductances associated with batteries according to their specific reversal potentials can be simulated instead (using the syn_tree function). The passive results were obtained in their purest form in dendrites without the associated somata and axons. Only for Figures $\mathbf{S 3}$ was the effect of somata explored in detail (see below).

\section{Passive dynamic responses to distributed synaptic conductances.}

Synaptic inputs were simulated as a Poisson process inducing synaptic conductances at a given frequency per synapse. The dynamics of the conductance trace was given by the form $G_{\text {syn }}=G_{\text {scale }}\left(e^{-\frac{t}{\tau_{1}}}-e^{-\frac{t}{\tau_{2}}}\right)$ with a rise time constant of $\tau_{2}=0.5 \mathrm{~ms}$ and a decay time constant of $\tau_{1}=2.5 \mathrm{~ms}$. $G_{\text {scale }}$ was set using Equation 1 to ensure that the integral over time of the synaptic conductance profile produced the same voltage as the steady state cases (compare Figure 2 rightmost panels with rightmost panels in Figure 3) at an input frequency of $5 \mathrm{~Hz}$ per synapse. Our novel TREES toolbox function LIF_tree was used without a voltage threshold for spiking in the case of the passive dynamic responses. LIF_tree injects distributed synapses into the conductance matrix that defines the dendritic tree in a timeresolved dynamic manner and produces local voltage responses throughout the dendrite. In Figures 3 and 4 the voltage time courses at the dendritic root were plotted for a subset of morphologies (the ones shown in Figure 3 and the first morphology in each of the 223 datasets in Figure 4) for better clarity.

\section{Effect of soma size on dendritic constancy - analytical treatment.}

Consider an electrotonically compact soma of radius $R$ attached to a dendritic cable of length $l \quad 500$ and radius $r$. The intrinsic properties are given by the specific conductance of the intracellular ${ }_{501}$ 
medium $G_{i}$ and membrane conductance $G_{m}$. The soma has a leak conductance of $G_{s}(R)=502$ $4 \pi R^{2} G_{m}$. The voltage along the cable due to a current injection of magnitude $I_{a p p}$ at the soma 503 is given by

$$
v(x)=\left[\frac{I_{a p p}}{G_{\infty}\left(1+G_{s}(R) \tanh \left(\frac{l}{\lambda}\right)\right)}\right] \frac{\cosh \left(\frac{l-x}{\lambda}\right)}{\cosh \left(\frac{l}{\lambda}\right)}
$$

for $\lambda=\sqrt{\frac{G_{i} d}{4 G_{m}}}$, the electrotonic length constant of the cable, and $G_{\infty}=\frac{\pi G_{i} d^{2}}{4 \lambda}$, the semi-infinite 505 conductance. Note that this derivation relies on a self-consistent description of the root voltage 506 $v_{0}$ due to the current flowing into the dendrite. Due to the symmetry of transfer resistance, 507 this is also the voltage induced at the soma by current injection at a site $x$. Consider the total 508 somatic response $V_{T o t}$ to distributed synaptic currents:

$$
\begin{gathered}
V_{\text {Tot }}=\int_{0}^{l} v(x) d x \\
V_{\text {Tot }}=\int_{0}^{l}\left[\frac{I_{a p p}}{G_{\infty}\left(1+G_{s}(R) \tanh \left(\frac{l}{\lambda}\right)\right)}\right] \frac{\cosh \left(\frac{l-x}{\lambda}\right)}{\cosh \left(\frac{l}{\lambda}\right)} d x \\
V_{\text {Tot }}=\frac{I_{\text {app }}}{\pi d G_{m}}\left[\frac{\tanh \left(\frac{l}{\lambda}\right)}{G_{s}(R)+\tanh \left(\frac{l}{\lambda}\right)}\right] .
\end{gathered}
$$

It can be seen that the term in brackets determines the deviation from dendritic constancy. 510 A small value of $G_{s}(R)$ is key as tanh is bounded by one. Figures $\mathbf{S 3}$ plots the relationship 511 between somatic radius $R$ and dendritic constancy for different electrotonic lengths $\frac{l}{\lambda}$ and the 512 relationship between the electrotonic length $\frac{l}{\lambda}$ and dendritic constancy for somatic radii $R$.

\section{Spiking model by Mainen and Sejnowski.}

We used our new tool T2N (Beining et al., 2017) to port the existing model for the spiking 515 mechanism by Mainen and Sejnowski (1996) \#2488 from ModelDB (Hines et al., 2004) to 516 our TREES toolbox package in Matlab. In T2N, calculating spike frequency vs. current 517 injections or vs. synaptic input frequencies using different dendritic morphologies becomes 518 easy to implement. The required simulations are distributed automatically on the available 519 computing cores and the entire toolset from the TREES toolbox becomes available to better 520 edit and analyse dendritic trees and the resulting simulation variables. The code is available 521 but, briefly, the simulations ran $40 \mathrm{sec}$ with a time step of $0.05 \mathrm{~ms}$ and a pre-run for $200 \mathrm{~ms}$. 522 
The initial voltage was set to $-70 \mathrm{mV}$, which was a close match for resting voltages for the ${ }_{523}$ four different morphologies. The voltage was calculated every $50 \mu m$ and a current injection 524 electrode was inserted into the root or synapse point processes into every node (separated 525 by $1 \mu \mathrm{m})$. Morphologies from the original model were translated into TREES toolbox and 526 resampled to $1 \mu \mathrm{m}$ internode distances. The dendritic diameters were normalised to $1 \mu m$ and 527 soma with axon divided into axon hillock, initial segment, nodes of Ranvier and myelinated 528 segments were added as in the original model with the respective ion channel conductances. 529 Implicit spines were modelled according to the original model for the current injections 530 but even the L3 aspiny cell was implemented as spiny in all cases for better comparison. 531 Responses to distributed synaptic inputs were modelled with $\operatorname{Exp} 2 S y n$ point processes with 532 rise time constant of $\tau_{2}=0.2 \mathrm{~ms}$ and decay time constant of $\tau_{1}=2.5 \mathrm{~ms}$ driven by NetStim 533 point processes in artificial point neurons under Poisson process conditions (noise 1) and 534 following a given input frequency. The random seeds for the NetSt im process were set to be 535 independent for different synapses.

\section{CA1 pyramidal cell spiking models.}

Electrotonic compartmentalisation and location dependent ion channel distributions allow for separate non-linear integration of inputs in different regions of dendrites. In order to check how these conditions affect our results, we studied two models of CA1 pyramidal cells that are known to produce dendritic spikes. The dendritic arborisation of pyramidal cells follows a laminar structure that generally reflects the different main excitatory afferents impinging on their dendrites from different brain regions. This distinctive structural organisation is also manifested in the way the electrotonic properties and active channels are distributed. Therefore, it was necessary to define how non-uniform channel distributions scale in the different morphologies.

We ported the model by Jarsky et al. (2005) to T2N in a similar manner as with the model by 548 Mainen and Sejnowski. This model includes four active conductances: a voltage-gated $\mathrm{Na}^{+}{ }_{549}$ conductance, a delayed rectifier $\mathrm{K}^{+}$conductance, a proximal A-type $\mathrm{K}^{+}$conductance, and a 550 distal A-type $\mathrm{K}^{+}$conductance with a higher half-inactivation voltage. These conductances 551 were distributed as a function of path distance from the soma. The $\mathrm{Na}^{+}$and the delayed 552 
rectifier $\mathrm{K}^{+}$conductance were modelled following a uniform distribution, the weak excitability ${ }_{553}$ version of the model by Jarsky. The A-type $\mathrm{K}^{+}$current was modelled with the experimentally ${ }_{554}$ reported six-fold increase in conductance along the apical dendrites resulting in variable ${ }_{555}$ slopes of the linear increase between soma and tuft in different morphologies. The apical 556 dendrites were divided with borders along the apical trunk to contain 3.14\% (proximal apical), 557 $36.27 \%$ (medial apical), $68.90 \%$ (distal) and $100 \%$ (tuft) of the total apical length respectively. ${ }_{558}$ These divisions occurred at path distances of around $100 \mu \mathrm{m}, 300 \mu \mathrm{m}$ and $500 \mu \mathrm{m}$.

\section{Poirazi et al. 2003 model.}

The model by Poirazi et al. (2003b) was also ported to T2N, and similarly adapted to apply ${ }_{561}$ to different pyramidal cell morphologies. The model consists of a wide variety of active and passive membrane mechanisms (see the online supplement in Poirazi et al. 2003b), including 17 types of ion channels, most of them non-uniformly distributed along the somato-dendritic axis. The apical trunk stems were divided according to laminar depth from soma to stratum lacunosum-moleculare ( $>68.90 \%$ from the total apical dendrite length, similarly as in the model by Jarsky) and the ion channel distributions were rescaled accordingly. The apical trunk dendrites that bifurcate within the stratum radiatum giving rise to two or more main apical dendrites were also considered as the apical trunk region. Similarly to the original Poirazi model, a peritrunk region was defined as the first $50 \mu \mathrm{m}$ in path length from every oblique branch that extended away from the apical trunk. The remaining apical branches were considered as the apical region with a further distinction of more distal dendrites, 572 located beyond a laminar depth away from the soma of $300 \mu m$ (distal apical) and $350 \mu m$ (tuft). 573 The passive parameters and channel densities were similar to the Poirazi model, except for 574 axial conductances being distributed uniformly and the leak reversal potential being fixed to $-70 \mathrm{mV}$ rendering slightly different resting potentials for each cell morphology.

\section{Integrate-and-fire spiking model with passive dendrite leak.}

Dynamic LIF spiking responses for all morphologies in Figures $2-4$ were obtained using the LIF_tree function in a similar way as for passive dynamic responses (see above). In the case of the LIF responses, synaptic conductances were set using Equation $\mathbf{1}^{\prime}$ to reach $-60 \mathrm{mV}$ at the dendrite root when activated at $1 \mathrm{~Hz}$. By then setting the voltage threshold of the LIF mechanism in the dendrite root to $-60 \mathrm{mV}$ we ensured that spiking started around $1 \mathrm{~Hz}$ input 

was reached at the dendritic root, resetting the voltage everywhere to $-70 \mathrm{mV}$. Morphologies from NeuroMorpho.Org were used in their pure dendritic form (without soma or axon) and after normalising dendritic diameters for each population.

\section{Adaptive exponential integrate-and-fire spiking model with passive den- ${ }^{587}$ drite leak.

Since the simple LIF is generally not able to reproduce the variety of temporal firing patterns that occur in real neurons we extended it by an adaptation current in combination with an 589 exponential activation term (Brette and Gerstner, 2005), while preserving passive parameters. 591 This also allowed us to test yet another spiking mechanism for our theory of dendritic constancy. Instead of a fixed threshold for spike initiation, action potentials in the adaptive exponential leaky integrate-and-fire (AdExpLIF) are generated through a positive, exponential feedback in the voltage of the dendritic root $V_{\text {root }}$, given by the differential equation $\frac{d V_{\text {root }}}{d t}=$ $\Delta_{T} \cdot e^{\left(\frac{V_{\text {root }}-V_{T}}{\Delta_{T}}\right)}$. By setting the slope factor to $\Delta_{T}=2 m V$ and the threshold to $V_{T}=-60 m V$ we made sure that spiking started around $1 \mathrm{~Hz}$ input frequency similar to our LIF simulations. The exponential activation term in the soma makes precise processing of fast fluctuating inputs during synaptic bombardment possible (Fourcaud-Trocmé et al., 2003), as spike initiation is not instantaneous in contrast to the LIF. The upswing of the potential beyond $-60 \mathrm{mV}$ grows rapidly to infinity, which is why the exact numerical threshold for a voltage reset has almost no influence on spike timing and was set to $V_{\text {thres }}=10 \mathrm{mV}$ in all simulations. Altering the parameters of spike initiation had no effects on the constancy of spike numbers with respect to morphology $(>50 \mathrm{mV})$. The adaptation current $w$ acted as a negative feedback on the voltage in each segment of the dendritic tree and was given by:

$$
\tau \frac{d w}{d t}=a\left(w-E_{L}\right)-w .
$$

Once the dendritic root reached $V_{\text {thres }}$, the voltage in each node was reset to $V_{\text {reset }}=-70 \mathrm{mV} \quad 606$ in the case of spike frequency adaptation. Increasing the reset voltage to $-60 \mathrm{mV}$ induced 607 bursting. After a spike was triggered, the variable $w$ was increased by an amount $b$ in all ${ }_{608}$ segments, which was $b=-60 f A$ in the adaptation and bursting neuron model. Depending on 609 $b$, the bursting neuron elicited several spikes in a short period of time until $w$ counterbalanced ${ }_{610}$ the exponential activation term, resulting in a longer ISI in between bursts. In case of the 611 
bursting model, the time constant was set to $30 \mathrm{~ms}$. Increasing the time constant to $\tau=100 \mathrm{~ms} \quad 612$ in combination with a high value of $b$ resulted in spike frequency adaptation.

\section{Stochastic inputs: Subthreshold voltage moments.}

Consider a sealed dendrite of physical length $l$ with electrotonic length constant $\lambda$. The voltage 615 time course at the proximal end due to a single brief injection of current of magnitude $a$ at electrotonic position $0 \leq x \leq l$ is given by

$$
\varepsilon(x, t)=\frac{a \lambda e^{-\frac{t}{\tau}}}{l}\left[\frac{1}{2}+\sum_{n=1}^{\infty} \cos \left(\frac{n \pi x}{l}\right) e^{-\left(\frac{n \pi \lambda}{l}\right)^{2} \frac{t}{\tau}}\right] .
$$

This is plotted in Figure $\mathbf{S 6} \mathbf{A}$ (dashed lines). Given that synapses are uniformly distributed over $[0, l]$, the expected (ensemble) value of $\varepsilon$ at a given time $t,\langle\varepsilon(t)\rangle$, is given by

$$
\langle\varepsilon(t)\rangle=\int_{0}^{L} \varepsilon(x, t) P[x] d x=\frac{a e^{-\frac{t}{\tau}}}{2 L}
$$

where we have written $L=\frac{l}{\lambda}$ for the electrotonic length. The voltage above rest at the soma, ${ }_{220}$ neglecting for the moment a threshold-rest mechanism, is given by a sum of independent ${ }_{621}$ synaptic inputs

$$
v(t)=\sum_{\left\{x_{i}, t_{i}\right\}} \varepsilon\left(x_{i}, t-t_{i}\right) \chi_{\left[t_{i}, \infty\right)}(t)
$$

where the times $t_{i}$ are given by a Poisson process of rate $r_{\lambda} l$, the locations $x_{i}$ are uniformly ${ }_{623}$ distributed along the dendrite, and $\chi_{\left[t_{i}, \infty\right)}(t)$ is the indicator function of the interval $\left[t_{i}, \infty\right) . \quad{ }_{624}$ The subthreshold steady-state mean voltage above rest $\langle v\rangle$ can be found by taking expectations ${ }_{625}$

$$
\langle v\rangle=r_{\lambda} L \int_{0}^{\infty} \frac{1}{L} \int_{0}^{L} \varepsilon(x, t) d x d t=\frac{a r_{\lambda} \tau}{2}
$$

This is independent of $L$. Similarly, the subthreshold variance in $v$ can be written as

$$
\operatorname{Var}(v)=r_{\lambda} L \int_{0}^{\infty} \frac{1}{L} \int_{0}^{L} \varepsilon^{2}(x, t) d x d t=\frac{a^{2} r_{\lambda} \tau}{8} \operatorname{coth}(L)
$$

where the $\operatorname{coth}(L)$ term approaches 1 in the limit of large $L$ (Figure $\mathbf{S 6} \mathbf{B}$, dashed lines). 


\section{Stochastic inputs: Subthreshold voltage moments for synaptic currents.}

The above calculations give the voltage impulse response at the soma. If a synapse has its own time course $\zeta(t)$ (with $\zeta(t)=0$ for $t<0$ ), then the somatic voltage above rest is given instead by

$$
v(t)=\sum_{\left\{x_{i}, t_{i}\right\}} \zeta * \varepsilon\left(x_{i}, t-t_{i}\right) \chi_{\left[t_{i}, \infty\right)}(t)
$$

where $\zeta * \varepsilon(x, t)=\int_{0}^{t} \zeta(\theta) \varepsilon(x, t-\theta) d \theta$ represents convolution in time. A typical synaptic filter ${ }_{632}$ is modelled as a difference of exponentials with timescales $\tau_{f}$ and $\tau_{s}$ such that

$$
\zeta(t)=\frac{e^{-\frac{t}{\tau_{f}}}-e^{-\frac{t}{\tau_{s}}}}{\tau_{f}-\tau_{s}}
$$

Note that each term in the series form of $\varepsilon(x, t)$ can be written as $c_{n} e^{-\frac{t}{\tau_{n}}}$ for some coefficient $c_{n}$ and timescale $\tau_{n}$ as defined above (with $c_{n}$ typically dependent on input location $x$ ). Then each such term convolves with $\zeta(t)$ to give

$$
\frac{c_{n} \tau_{n}}{\tau_{s}-\tau_{f}}\left(e^{-\frac{t}{\tau_{n}}}\left(\frac{\tau_{f}}{\tau_{f}-\tau_{n}}-\frac{\tau_{s}}{\tau_{s}-\tau_{n}}\right)-\left(\frac{\tau_{f} e^{-\frac{t}{\tau_{f}}}}{\tau_{f}-\tau_{n}}-\frac{\tau_{s} e^{-\frac{t}{\tau_{s}}}}{\tau_{s}-\tau_{n}}\right)\right)
$$

if $\tau_{f}, \tau_{s} \neq \tau_{n}$. In the case that one of $\tau_{f}=\tau_{n}$ or $\tau_{s}=\tau_{n}$ (without loss of generality let $\tau_{f}=\tau_{n}$ ) ${ }^{637}$ the form is instead

$$
\frac{c_{n}}{\tau_{s}-\tau_{n}}\left(\left(\frac{\tau_{s} \tau_{n}}{\tau_{s}-\tau_{n}}\right)\left(e^{-\frac{t}{\tau_{n}}}-e^{-\frac{t}{\tau_{s}}}\right)-t e^{-\frac{t}{\tau_{n}}}\right)
$$

with an additional synaptic filter alongside the dendritic filter given by Equation 11, the 639 difference in somatic voltage responses to proximal and distal inputs is reduced even for 640 synapses that are fast compared to the membrane time constant (Figure S6A).

The subthreshold mean is unchanged from the instantaneous case as $\int_{0}^{\infty} \zeta(\theta) d \theta=1$, and the 642 subthreshold variance can be computed by squaring the above terms, integrating $t$ from 0 to 643 $\infty$ and $x$ from 0 to $l$, and summing the infinite series. The result is cumbersome to write in 644 full, but can be plotted in Figure S6B. The variance is lower in the case of the synaptic filter 645 compared to instantaneous current injection. 
Cuntz et al.

\section{Stochastic inputs: Subthreshold characteristic functions and firing rate ap- ${ }_{647}$ proximation.

The firing rate can in principle be calculated exactly from the expected time for the stochastic 649 process (Equation 13) to first reach the firing threshold $v_{t h}$ from the voltage reset $v_{r e}$. Given 650 a uniform initial voltage $v_{0}$ (which decays with timescale $\tau$ ), the random variable $V_{\left\{T, v_{0}\right\}} \quad{ }^{651}$ describes the voltage $T$ seconds later. The characteristic function $\phi_{v}\left(s, T, v_{0}\right)=\mathbb{E}\left[e^{-s V_{\left\{T, v_{0}\right\}}}\right]$ of ${ }_{652}$ $V_{\left\{T, v_{0}\right\}}$ is given by (Rice, 1944)

$$
\phi_{v}\left(s, T, v_{0}\right)=\exp \left[-r l\left(\int_{0}^{T} 1-\mathbb{E}_{x}\left[e^{-s[\varepsilon(x, t)]}\right] d t\right)+v_{0} e^{-\frac{T}{\tau}}\right]
$$

where the expectation $\mathbb{E}_{x}$ is over synaptic locations $x$. This can be inverted to give the 654 probability distribution $f_{v}$ of $V_{\left\{T, v_{0}\right\}}$. An additional integral transform over $T, \psi_{v}\left(\rho, v_{0}\right)={ }_{655}$ $\mathbb{E}\left[e^{-\rho T} f_{v}\right]$, allows the moment generating function $M_{F P}(t)$ of the first-passage time density ${ }_{656}$ to be written as (Siegert, 1951)

$$
M_{F P}(t)=\frac{\psi_{v}\left(-\rho, v_{r e}\right)}{\psi_{v}\left(-\rho, v_{t h}\right)}
$$

The mean first-passage time, and hence the output firing rate, could then be extracted from 658 $\left.\frac{d M_{F P}}{d t}\right|_{t=0}$.

In practice, the above procedure is numerically sensitive and the following approximation is robust to the high cumulative input rates typically seen across an entire dendritic tree. Taking the subthreshold voltage mean $\mu_{v}$ and standard deviation $\sigma_{v}$ allows the firing rate $R$ to be accurately approximated (Alijani and Richardson, 2011) using the equation from Brunel and Hakim (1999)

$$
R^{-1}=\tau \int_{0}^{\infty} \frac{1}{z} e^{-\frac{z^{2}}{2}}\left(e^{z z_{t h}}-e^{z z_{r e}}\right) d z
$$

where $z_{t h}=\frac{v_{t h}-\mu_{v}}{\sigma_{v}}$ and $z_{r e}=\frac{v_{r e}-\mu_{v}}{\sigma_{v}}$. This is plotted as a function of dendrite length in 665 Figure $\mathbf{S 6} \mathrm{B}$ and as a function of input firing rate in Figure $\mathrm{S6} \mathrm{C}$.

Combining the above equations, the output firing rate $R$ can be written, in the case of instan- 667 taneous synapses, in terms of intrinsic quantities as 


$$
R^{-1}=\frac{C}{G_{m}} \int_{0}^{\infty} \frac{1}{z} e^{-\frac{z^{2}}{2}}\left[e^{z \sqrt{\frac{2 \lambda G_{m}}{r l C \operatorname{coth} \frac{l}{\lambda}}}\left(\frac{2 \pi d G_{m} v_{t h}}{I_{d i s t}}-\frac{r l C}{\lambda G_{m}}\right)}-e^{z \sqrt{\frac{2 \lambda G_{m}}{r l C \operatorname{coth} \frac{l}{\lambda}}}\left(\frac{2 \pi d G_{m} v_{r e}}{I_{d i s t}}-\frac{r l C}{\lambda G_{m}}\right)}\right] d z
$$

where, as before, $C$ is the specific capacitance, $G_{m}$ is the membrane conductivity, $l$ is the 669 dendrite length, $d$ is the average diameter, $\lambda=\sqrt{\frac{G_{i} d}{4 G_{m}}}$ is the electrotonic length, $G_{i}$ is the axial 670 conductivity, and $I_{\text {dist }}$ is the current induced by a single synapse. Additionally, $r$ is the rate of 671 synaptic activation per $\mu m$, and $v_{r e}$ and $v_{t h}$ are the reset and threshold voltages respectively. $\quad 672$

In the case of filtered synapses, there is not a compact form for $R$ and Equation 22 is used 673 directly with the subthreshold mean and variance as derived above. The code to calculate 674 $R$ analytically can be found in the function $L I F_{-} F R_{-} t r e e$ for synaptically filtered current 675 injections.

\section{References}

Adachi R, Yamada R, Kuba H (2015) Plasticity of the axonal trigger zone. Neuroscien- 678 tist 21:255-265.

Alijani AK, Richardson MJE (2011) Rate response of neurons subject to fast or frozen noise: 680 From stochastic and homogeneous to deterministic and heterogeneous populations. Physical ${ }_{681}$ Review E 84:011919.

Ascoli GA (2006) Mobilizing the base of neuroscience data: the case of neuronal morphologies. 683 Nature Reviews Neuroscience 7:318-324.

Bakken TE, Stevens CF (2011) Visual system scaling in teleost fish. Journal of Comparative Neurology 153:142-153.

Beining M, Mongiat LA, Schwarzacher SW, Cuntz H, Jedlicka P (2017) T2N as a new tool for robust electrophysiological modeling demonstrated for mature and adult-born dentate granule cells. eLife 6:e26517.

Bekkers JM, Stevens CF (1990) Two different ways evolution makes neurons larger. Progress in Brain Research 83:37-45. 
Bird AD, Cuntz H (2016) Optimal current transfer in dendrites. PLOS Computational Biol- 692 ogy 12:e1004897.

Borst A, Haag J (1996) The intrinsic electrophysiological characteristics of fly lobula plate tan- 694 gential cells: I. Passive membrane properties. Journal of Computational Neuroscience 3:313-336. 695

Branco T, Clark BA, Häusser M (2010) Dendritic discrimination of temporal input sequences 696 in cortical neurons. Science 329:1671-1675.

Brette R, Gerstner W (2005) Adaptive exponential integrate-and-fire model as an effective 698 description of neuronal activity. Journal of Neurophysiology 94:3637-3642.

Brown CE, Boyd JD, Murphy TH (2010) Longitudinal in vivo imaging reveals balanced 700 and branch-specific remodeling of mature cortical pyramidal dendritic arbors after stroke. 701 Journal of Cerebral Blood Flow and Metabolism 30:783-791.

Brunel N, Hakim V (1999) Fast global oscillations in networks of integrate-and-fire neurons 703 with low firing rates. Neural Computation 11:1621-1671.

Carnevale NT, Hines ML (2004) The NEURON Book. Cambridge University Press.

Chavlis S, Petrantonakis PC, Poirazi P (2017) Dendrites of dentate gyrus granule cells con- 706 tribute to pattern separation by controlling sparsity. Hippocampus 27:89-110.

Chen X, Yuan LL, Zhao C, Birnbaum SG, Frick A, Jung WE, Schwarz TL, Sweatt JD, Johnston 708 D (2006) Deletion of Kv4.2 gene eliminates dendritic A-type K+ current and enhances 709 induction of long-term potentiation in hippocampal CA1 pyramidal neurons. Journal of 710 Neuroscience 26:12143-12151.

Connelly WM, Crunelli V, Errington AC (2016) Passive synaptic normalization and input 712 synchrony-dependent amplification of cortical feedback in thalamocortical neuron dendrites. 713 Journal of Neuroscience 36:3735-3754.

Conrad CD, Ortiz JB, Judd JM (2017) Chronic stress and hippocampal dendritic complexity: 715 Methodological and functional considerations. Physiology \& Behavior 178:66-81. Functional consequences of age-related morphologic changes to pyramidal neurons of the 718 rhesus monkey prefrontal cortex. Journal of Computational Neuroscience 38:263-283. 
Cuntz H, Forstner F, Borst A, Häusser M (2010) One rule to grow them all: a general theory of 720 neuronal branching and its practical application. PLoS Computational Biology 6:e1000877. 721

Cuntz H, Forstner F, Haag J, Borst A (2008) The morphological identity of insect dendrites. 722 PLoS Computational Biology 4:e1000251.

Cuntz H, Forstner F, Schnell B, Ammer G, Raghu SV, Borst A (2013) Preserving neural function 724 under extreme scaling. PLOS ONE 8:e71540.

Cuntz H, Haag J, Borst A (2003) Neural image processing by dendritic networks. 726 PNAS 100:11082-11085.

Cuntz H, Haag J, Forstner F, Segev I, Borst A (2007) Robust coding of flow-field parameters 728 by axo-axonal gap junctions between fly visual interneurons. PNAS 104:10229-10233. 729

Cuntz H, Mathy A, Häusser M (2012) A scaling law derived from optimal dendritic wiring. 730 PNAS 109:11014-11018.

Denève S, Alemi A, Bourdoukan R (2017) The brain as an efficient and robust adaptive learner. 732 Neuron 94:969-977.

Denève S, Machens CK (2016) Efficient codes and balanced networks. Nature Neuro- 734 science 19:375-382.

Einstein G, Buranosky R, Crain BJ (1994) Dendritic pathology of granule cells in Alzheimer's 736 disease is unrelated to neuritic plaques. Journal of Neuroscience 14:5077-5088. ${ }_{737}$

Evans MD, Dumitrescu AS, Kruijssen DL, Taylor SE, Grubb MS (2015) Rapid modulation of 738 axon initial segment length influences repetitive spike firing. Cell Reports 13:1233-1245. 739

Fourcaud-Trocmé N, Hansel D, van Vreeswijk C, Brunel N (2003) How spike generation 740 mechanisms determine the neuronal response to fluctuating inputs. Journal of Neuro- 741 science 23:11628-11640.

Gabbiani F, Krapp HG, Koch C, Laurent G (2002) Multiplicative computation in a visual 743 neuron sensitive to looming. Nature 420:320-324.

Garden DLF, Dodson PD, O'Donnell C, White MD, Nolan MF (2008) Tuning of Synaptic 745 Integration in the Medial Entorhinal Cortex to the Organization of Grid Cell Firing Fields. 746 Neuron 60:875-889. 
Gidon A, Segev I (2012) Principles governing the operation of synaptic inhibition in dendrites. 748 Neuron 75:330-341.

Gjorgjieva J, Drion G, Marder E (2016) Computational implications of biophysical diversity 750 and multiple timescales in neurons and synapses for circuit performance. Current Opinion 751 in Neurobiology 37:44-52.

Gulledge AT, Bravo JJ (2016) Neuron morphology influences axon initial segment plasticity. 753 eneuro 3:ENEURO.0085-15.2016.

Häusser M (2001) Synaptic function: dendritic democracy. Current Biology 11:R10-12.

Hines ML, Morse T, Migliore M, Carnevale NT, Shepherd GM (2004) ModelDB: A database to 756 support computational neuroscience. Journal of Computational Neuroscience 17:7-11. 757

Jaffe DB, Carnevale NT (1999) Passive normalization of synaptic integration influenced by 758 dendritic architecture. Journal of Neurophysiology 82:3268-3285. 759

Jarsky T, Roxin A, Kath WL, Spruston N (2005) Conditional dendritic spike propagation 760 following distal synaptic activation of hippocampal CA1 pyramidal neurons. Nature Neuro- 761 science 8:1667-1676.

Koch C, Douglas RJ, Wehmeier U (1990) Visibility of synaptically induced conductance 763 changes: theory and simulations of anatomically characterized cortical pyramidal cells. 764 Journal of Neuroscience 10:1728-1744. 765

Koch C, Segev I (1999) Methods in neuronal modeling - from ions to networks MIT Press, 766 Cambridge, MA.

Kuba H (2012) Structural tuning and plasticity of the axon initial segment in auditory neurons. 768 Journal of Physiology 590:5571-5579.

Kuba H, Oichi Y, Ohmori H (2010) Presynaptic activity regulates Na+ channel distribution at 770 the axon initial segment. Nature 465:1075-1078.

Leal SL, Yassa MA (2013) Perturbations of neural circuitry in aging, mild cognitive impairment, 772 and Alzheimer's disease. Ageing Research Reviews 12:823-831.

London M, Häusser M (2005) Dendritic computation. Annual Review of Neuroscience 28:503-532. 774 
London M, Meunier C, Segev I (1999) Signal transfer in passive dendrites with nonuniform 775 membrane conductance. Journal of Neuroscience 19:8219-8233.

London M, Roth A, Beeren L, Häusser M, Latham PE (2010) Sensitivity to perturbations in 777 vivo implies high noise and suggests rate coding in cortex. Nature 466:123-127.

London M, Segev I (2001) Synaptic scaling in vitro and in vivo. Nature Neuroscience 4:853-855. 779

Luebke JI, Medalla M, Amatrudo JM, Weaver CM, Crimins JL, Hunt B, Hof PR, Peters A (2015) 780 Age-related changes to layer 3 pyramidal cells in the rhesus monkey visual cortex. Cerebral ${ }_{781}$ Cortex 25:1454-1468.

Magee (1999) Dendritic Ih normalizes temporal summation in hippocampal CA1 neurons. 783 Nature Neuroscience 2:508-514.

Magee JC (2000) Dendritic integration of excitatory synaptic input. Nature Reviews Neuro- 785 science 1:181-190.

Magee JC, Cook EP (2000) Somatic EPSP amplitude is independent of synapse location in 787 hippocampal pyramidal neurons. Nature Neuroscience 3:895-903.

Mainen ZF, Joerges J, Huguenard JR, Sejnowski TJ (1995) A model of spike initiation in 789 neocortical pyramidal neurons. Neuron 15:1427-1439.

Mainen ZF, Sejnowski TJ (1996) Influence of dendritic structure on firing pattern in model neocortical neurons. Nature 382:363-366.

Markram H, Muller E, Ramaswamy S, Reimann MW, Abdellah M, Sanchez CA, Ailamaki 793 A, Alonso-Nanclares L, Antille N, Arsever S, Kahou GAA, Berger TK, Bilgili A, Buncic N, 794 Chalimourda A, Chindemi G, Courcol JD, Delalondre F, Delattre V, Druckmann S, Dumusc 795 R, Dynes J, Eilemann S, Gal E, Gevaert ME, Ghobril JP, Gidon A, Graham JW, Gupta A, 796 Haenel V, Hay E, Heinis T, Hernando JB, Hines M, Kanari L, Keller D, Kenyon J, Khazen G, 797 Kim Y, King JG, Kisvarday Z, Kumbhar P, Lasserre S, Le Bé JV, Magalhães BRC, Merchán- 798 Pérez A, Meystre J, Morrice BR, Muller J, Muñoz-Céspedes A, Muralidhar S, Muthurasa K, 799 Nachbaur D, Newton TH, Nolte M, Ovcharenko A, Palacios J, Pastor L, Perin R, Ranjan R, 800 Riachi I, Rodríguez JR, Riquelme JL, Rössert C, Sfyrakis K, Shi Y, Shillcock JC, Silberberg G, 801 Silva R, Tauheed F, Telefont M, Toledo-Rodriguez M, Tränkler T, Van Geit W, Díaz JV, Walker 802 
Mckay BE, Turner RW (2005) Physiological and morphological development of the rat 805 cerebellar Purkinje cell. Journal of Physiology 567:829-850.

Platschek S, Cuntz H, Deller T, Jedlicka P (2017) Lesion-induced dendritic remodeling as a 807 new mechanism of homeostatic structural plasticity in the adult brain In The Rewiring Brain, 808 Vol. 15, pp. 203-218. Elsevier.

Platschek S, Cuntz H, Vuksic M, Deller T, Jedlicka P (2016) A general homeostatic principle 810 following lesion induced dendritic remodeling. Acta Neuropathologica Communications 4:19. 811

Poirazi P, Brannon T, Mel BW (2003a) Arithmetic of subthreshold synaptic summation in a 812 model CA1 pyramidal cell. Neuron 37:977-987.

Poirazi P, Brannon T, Mel BW (2003b) Pyramidal neuron as two-layer neural network. Neu- 814 ron 37:989-999.

Polsky A, Mel BW, Schiller J (2004) Computational subunits in thin dendrites of pyramidal 816 cells. Nature Neuroscience 7:621-627.

Qin L, Jing D, Parauda S, Carmel J, Ratan RR, Lee FS, Cho S (2014) An adaptive role for 818 BDNF Val66Met polymorphism in motor recovery in chronic stroke. Journal of Neuro- 819 science 34:2493-2502.

Rall W (1959) Branching dendritic trees and motoneuron membrane resistivity. Experimental 821 Neurology 527:491-527.

Rall W (1962) Theory of physiological properties of dendrites. Annals of the New York Academy 823 of Sciences 96:1071-1092.

Rall W, Burke RE, Smith TG, Nelson PG, Frank K (1967) Dendritic location of synapses and 825 possible mechanisms for the monosynaptic EPSP in motoneurons. Journal of Neurophysiol- 826 ogy 30:1169-1193.

Rall W, Rinzel J (1973) Branch input resistance and steady attenuation for input to one branch 828 of a dendritic neuron model. Biophysical Journal 13:648-687.

Rice SO (1944) Mathematical analysis of random noise. Bell Systems . 
Rihn LL, Claiborne BJ (1990) Dendritic growth and regression in rat dentate granule cells ${ }_{831}$ during late postnatal development. Developmental Brain Research 54:115-124.

Rinzel J, Rall W (1974) Transient response in a dendritic neuron model for current injected at 833 one branch. Biophysical journal 14:759-790.

Rudolph M, Destexhe A (2003) A fast-conducting, stochastic integrative mode for neocortical ${ }_{835}$ neurons in vivo. Journal of Neuroscience 23:2466-2476.

Rushton WAH (1937) Initiation of the propagated disturbance. Proceedings of the Royal Society ${ }_{837}$ B 124:210-243.

Schmidt-Hieber C, Jonas P, Bischofberger J (2007) Subthreshold dendritic signal processing and 839 coincidence detection in dentate gyrus granule cells. Journal of Neuroscience 27:8430-8441. $\quad 840$

Schmidt-Hieber C, Nolan MF (2017) Synaptic integrative mechanisms for spatial cognition. ${ }_{841}$ Nature Neuroscience 20:1483-1492.

Segev I, London M (2000) Untangling dendrites with quantitative models. Science 290:744-750. 843

Siegert AJF (1951) On the First Passage Time Probability Problem. Physical Review 81:617-623. 844

Single S, Borst A (1998) Dendritic integration and its role in computing image velocity. 845 Science 281:1848-1850.

Šišková Z, Justus D, Kaneko H, Friedrichs D, Henneberg N, Beutel T, Pitsch J, Schoch S, ${ }_{847}$ Becker A, von der Kammer H, Remy S (2014) Dendritic structural degeneration is func- 848 tionally linked to cellular hyperexcitability in a mouse model of Alzheimer's disease. Neu- ${ }^{849}$ ron 84:1023-1033.

Snider J, Pillai A, Stevens CF (2010) A universal property of axonal and dendritic arbors. ${ }^{851}$ Neuron 66:45-56.

Spires TL, Hyman BT (2004) Neuronal structure is altered by amyloid plaques. Reviews in the 853 Neurosciences 15:267-278.

Stein RB (1965) A theoretical analysis of neuronal variability. Biophysical Journal 5:173-194. $\quad 855$

Steward O, Vinsant SL, Davis L (1988) The process of reinnervation in the dentate gyrus 856 of adult rats: an ultrastructural study of changes in presynaptic terminals as a result of ${ }_{857}$ sprouting. Journal of Comparative Neurology 267:203-210. 
Teeter CM, Stevens CF (2011) A general principle of neural arbor branch density. Current ${ }^{859}$ Biology 21:2105-2108.

Turrigiano GG (2017) The dialectic of Hebb and homeostasis. Philosophical transactions of the 861 Royal Society of London. Series B, Biological sciences 372:4-6.

Turrigiano GG, Nelson SB (2004) Homeostatic plasticity in the developing nervous system. ${ }_{863}$ Nature Reviews Neuroscience 5:97-107.

van Elburg RAJ, van Ooyen A (2010) Impact of dendritic size and dendritic topology on burst 865 firing in pyramidal cells. PLoS Computational Biology 6:e1000781.

van Ooyen A, Duijnhouwer J, Remme MWH, van Pelt J (2002) The effect of dendritic topology 867 on firing patterns in model neurons. Network: Computation in Neural Systems 13:311-325. 868

Vetter P, Roth A, Häusser M (2001) Propagation of action potentials in dendrites depends on 869 dendritic morphology. Journal of Neurophysiology 85:926-937.

Vuksic M, Del Turco D, Vlachos A, Schuldt G, Müller CM, Schneider G, Deller T (2011) Unilat- ${ }_{871}$ eral entorhinal denervation leads to long-lasting dendritic alterations of mouse hippocampal 872 granule cells. Experimental Neurology 230:176-185.

Williams SR, Stuart GJ (2003) Role of dendritic synapse location in the control of action 874 potential output. Trends in Neurosciences 26:147-154.

Yassa MA, Muftuler LT, Stark CEL (2010) Ultrahigh-resolution microstructural diffusion tensor 876 imaging reveals perforant path degradation in aged humans in vivo. PNAS 107:12687-12691. 877 


\section{Supporting information}
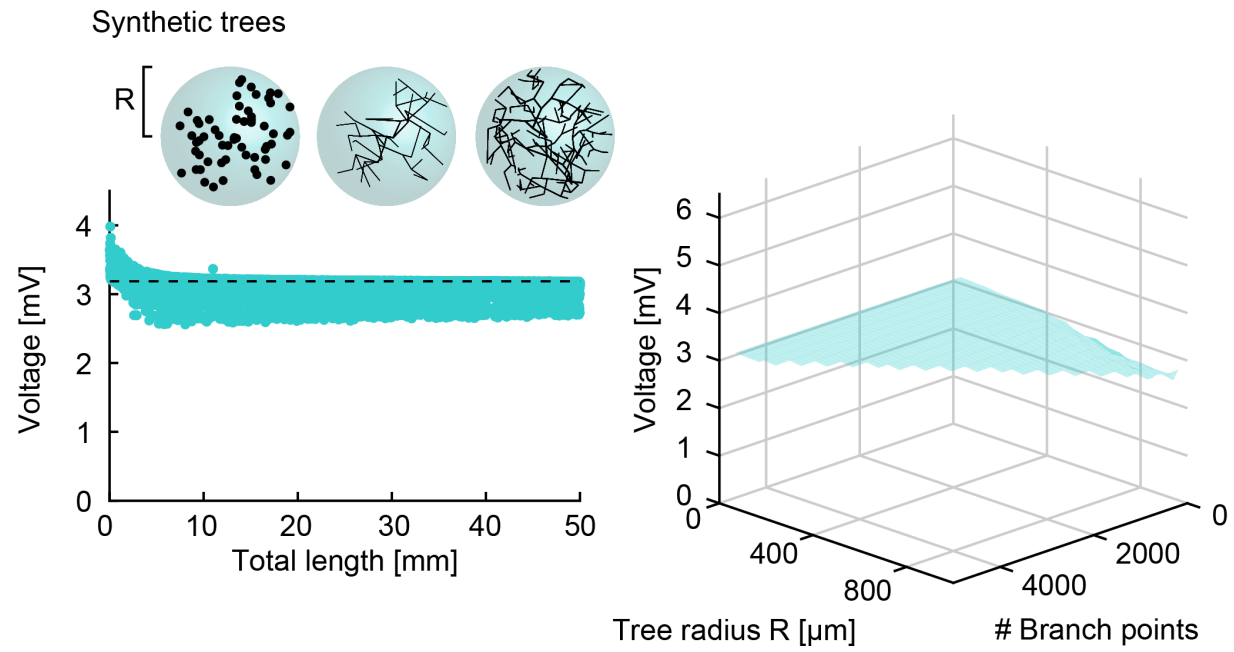

Fig S1. Steady-state passive responses to distributed inputs in synthetic dendrites are independent of dendrite length and shape.

Similar analysis as in Figure 4 but for 10,000 synthetic dendritic trees obtained using extended minimum spanning trees that reproduce many features of real dendrites (Cuntz et al., 2010). These cover a wide range of tree complexities as well as overall sizes (see Methods). 


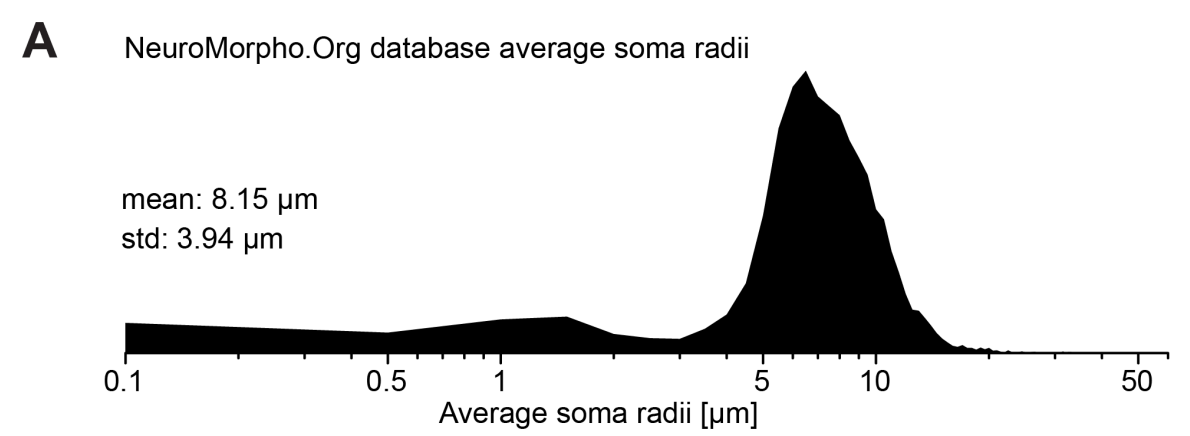

B NeuroMorpho.Org database average dendrite diameters

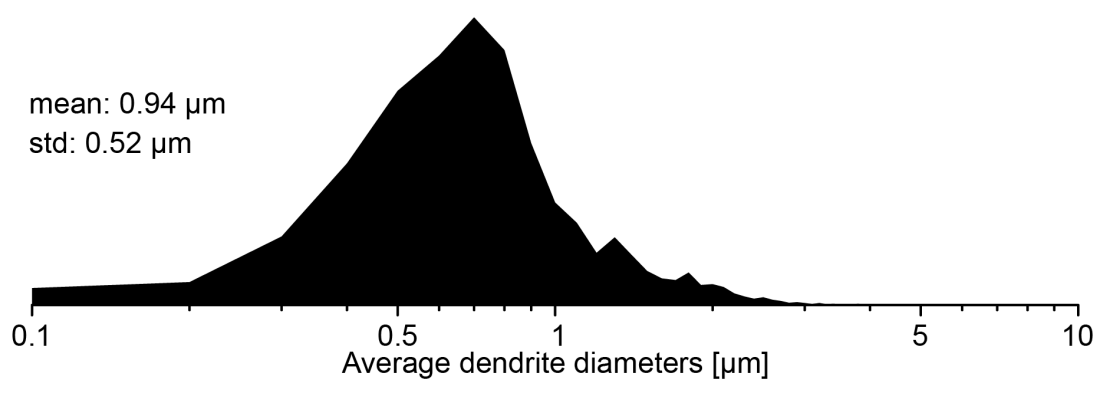

Fig S2. Distributions of diameters in somata and dendrites of the NeuroMorpho.Org database.

A, Distribution of soma radii in NeuroMorpho.Org. Not every cell had well-reconstructed somata explaining the tail of very small radii. High-quality soma reconstructions were not an inclusion criterion for this study that focuses on dendritic trees in the main text. B, Distribution of average dendrite diameters after resampling to $1 \mu \mathrm{m}$ internode distances to weigh each location in the dendritic tree homogeneously. 
bioRxiv preprint doi: https://doi.org/10.1101/787911; this version posted October 1,2019 . The copyright holder for this preprint (which was not certified by peer review) is the author/funder, who has granted bioRxiv a license to display the preprint in perpetuity. It is made available under aCC-BY-NC-ND 4.0 International license.

A Adding somata, not included in diameter normalisation
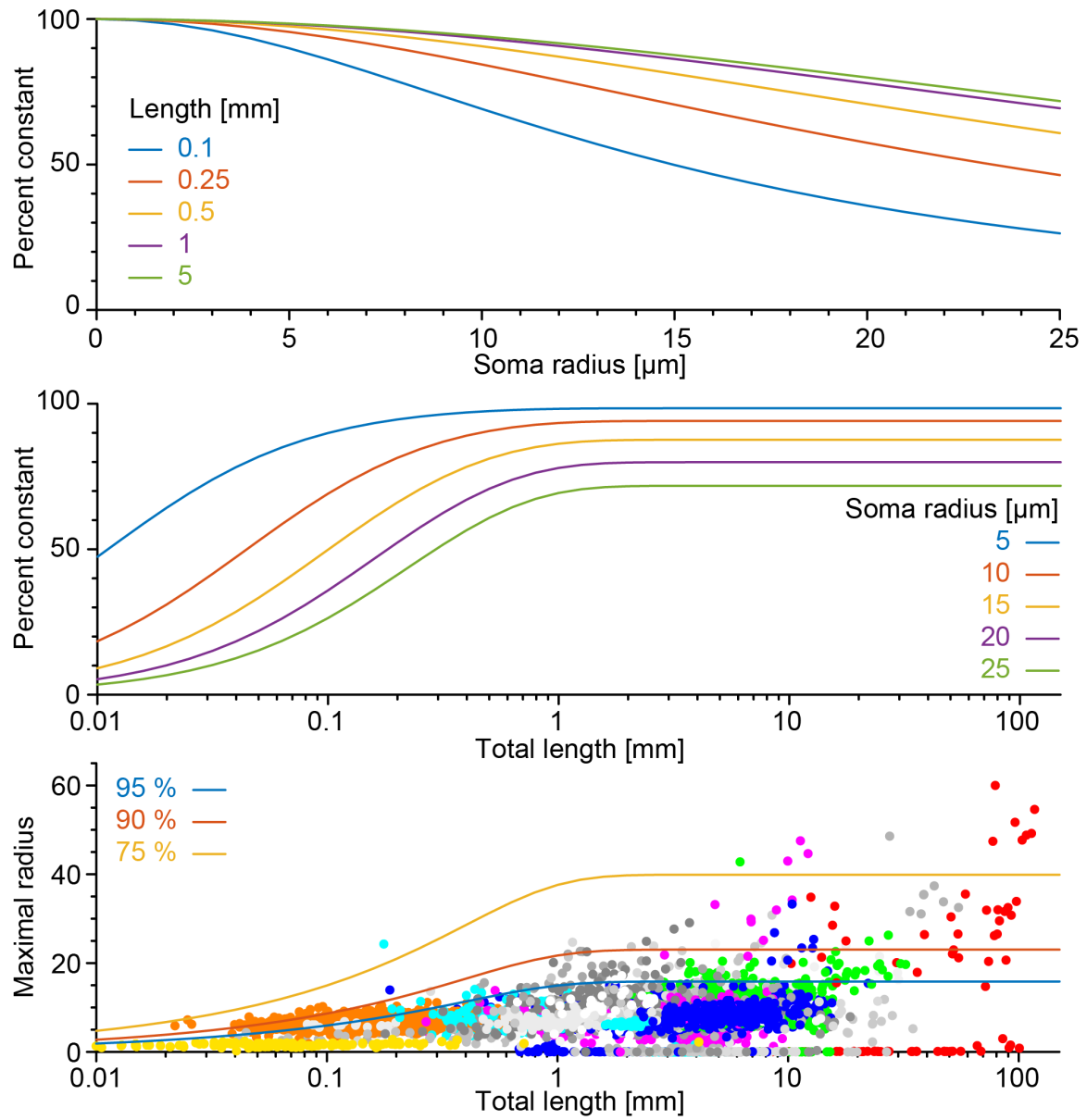

B NeuroMorpho.Org database with somata included in diameter normalisation
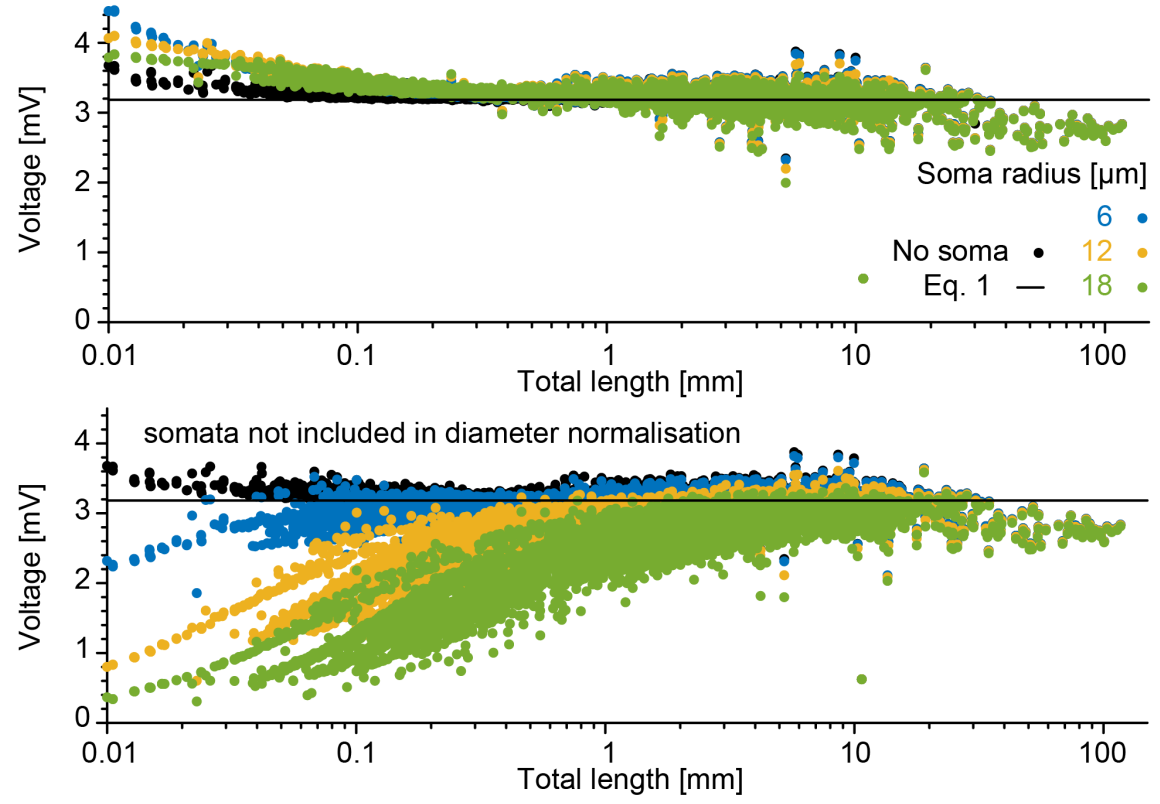

Fig S3. Effect of soma size on dendritic constancy.

See next page 
Fig S3. (continued) A, Analytical calculations of relative deviation from dendritic constancy (compared to $100 \%$ ) as a function of somatic radius for different lengths of dendrite (top panel) and as a function of length for different somatic radii (bottom panels). Used cables had $1 \mu \mathrm{m}$ diameter, specific membrane conductance $G_{m}=50 \frac{\mu S}{\mathrm{~cm}^{2}}$ and specific axial resistance of $R i=100 \Omega \mathrm{cm}$. B, Similar calculation for the NeuroMorpho.Org database as Figure $4 \mathrm{~A}$ but with appended somata that do not receive synaptic inputs (see Methods for more details). Here, colours indicate the radius of the appended soma. 
Inhomogeneous synaptic weights

A Jarsky et al. model

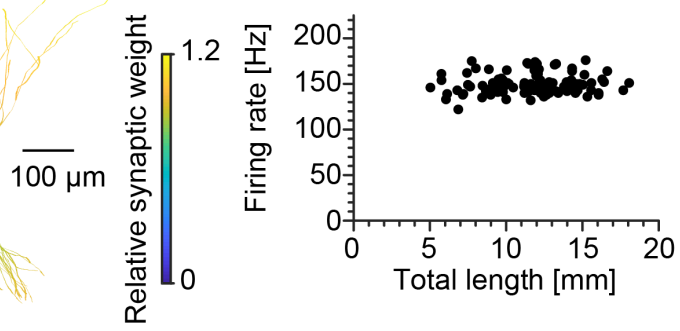

B LIF NeuroMorpho.Org dendrites
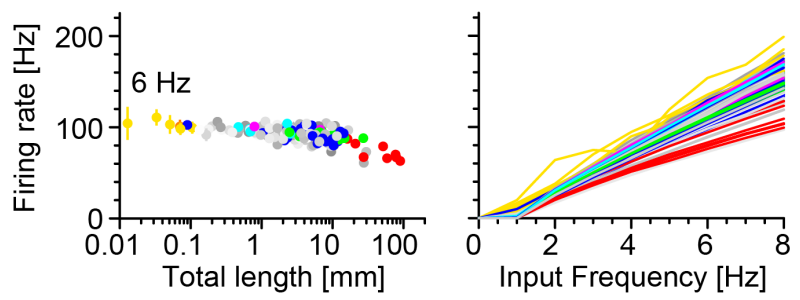

Fig S4. Effect of inhomogeneous distance-dependent synapse weights on dendritic constancy.

In these plots, synapses were scaled from $0.8 \times$ to $1.2 \times$ in a linear relation with distance (path length) from soma. A, Analogous to Figure $6 \mathbf{B}$, the model by Jarsky et al. (2005) with inhomogeneous synapse weights. B, Analogous to Figure 8A, the LIF model in NeuroMorpho.Org morphologies with inhomogeneous synapse weights. 
A Jarsky et al. model

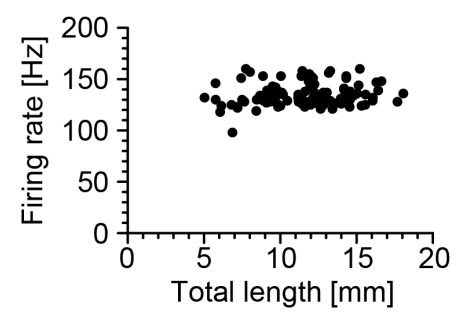

B LIF NeuroMorpho.Org dendrites
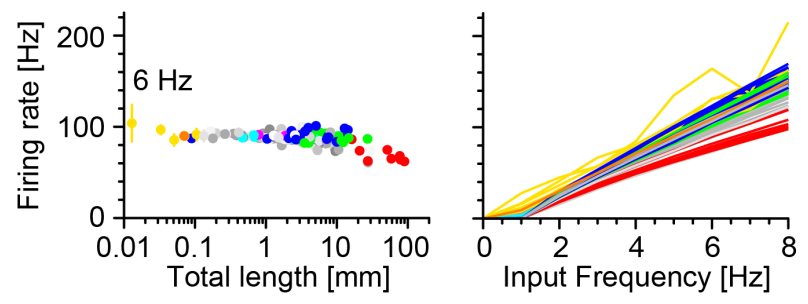

\section{Fig S5. Effect of inhibitory synapses on dendritic constancy.}

In these plots, $20 \%$ of all synapses were randomly selected to have a reversal potential of $-80 \mathrm{mV}$, which renders them inhibitory synapses. A, Analogous to Figure 6B, the model by Jarsky et al. (2005) with 20\% inhibitory synapses. B, Analogous to Figure 8A, the LIF model in NeuroMorpho.Org morphologies with $20 \%$ inhibitory synapses. 
Analytical calculations in the cable: Voltage to spike transformation

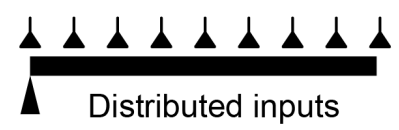

A Time course

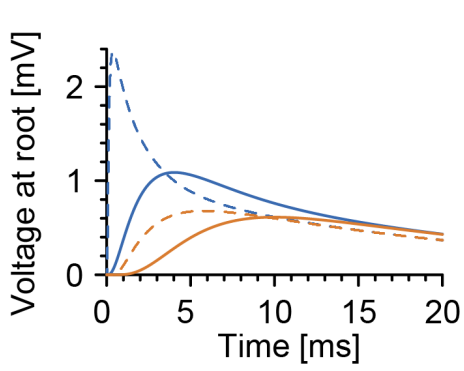

Synaptic filter at

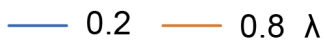

Instantaneous at

$---0.2--0.8 \lambda$

B Length invariance

C Input-output function

Input firing rate $[\mathrm{kHz} / \lambda]$
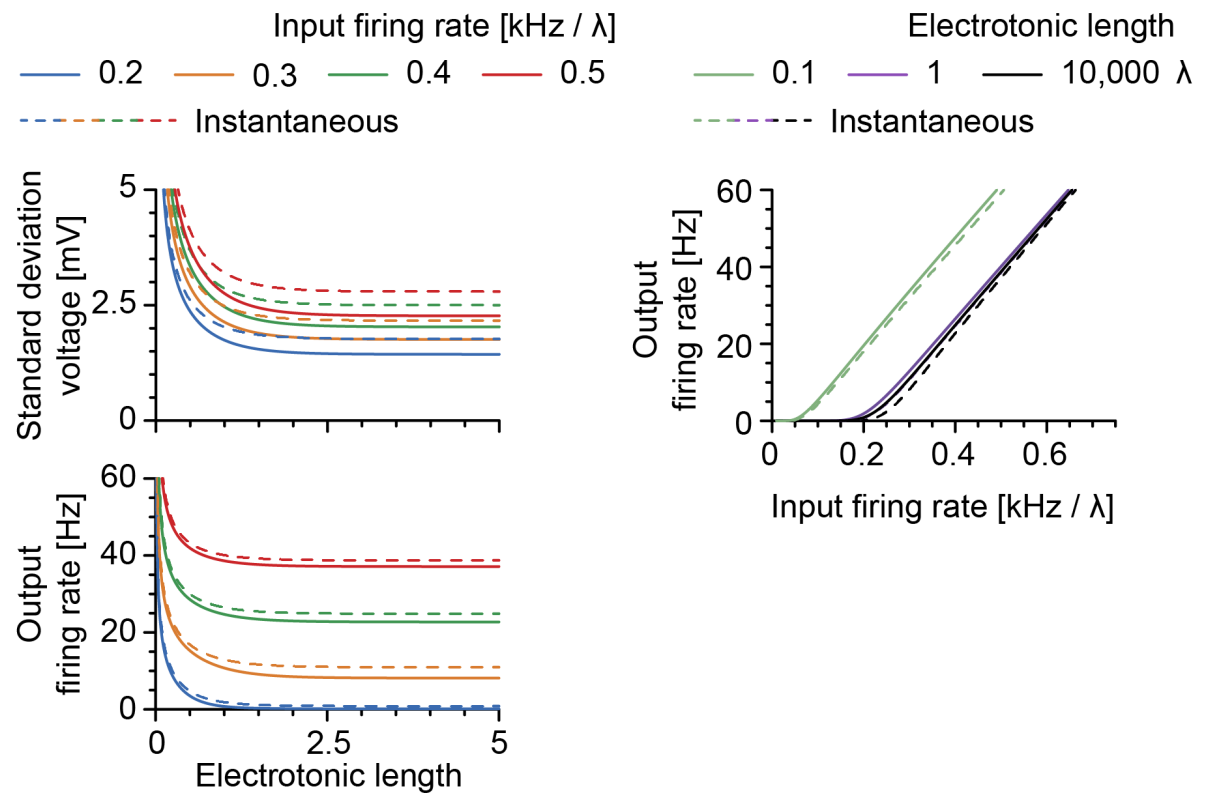

Fig S6. Transformation of voltage fluctuations into spikes.

A, Voltage response as a function of time at the proximal end of a sealed dendrite of electrotonic length 1 to an instantaneous (dashed lines) and synaptically filtered (double exponential $\tau_{\text {rise }}=0.5 \mathrm{~ms}, \tau_{\text {decay }}=2.5 \mathrm{~ms}$, solid lines) current injection with magnitude $a=1$. Blue lines show responses at 0.2 and yellow lines at 0.8 electrotonic distance. The time constant of the membrane was $\tau=20 \mathrm{~ms}$. B, Top panel, subthreshold proximal voltage variance as a function of electrotonic length for different input rates per unit electrotonic length: 200, 300,400, and $500 \mathrm{~Hz} . \quad a=2.5$ and $\tau=20 \mathrm{~ms}$. Bottom panel, firing rate as a function of electrotonic length for different input rates per unit electrotonic length: 200, 300, 400, and 500 Hz. $a=2.5$ and $\tau=20 \mathrm{~ms}$. As in $\mathbf{A}$, dashed lines for instantaneous and solid lines for filtered current injections. C, Output firing rate as a function of afferent rate for dendrites of different electrotonic lengths: $0.1,1$, and 10,000. $a=2.5$ and $\tau=20 \mathrm{~ms}$. As in A, dashed lines for instantaneous and solid lines for filtered current injections. 
Table S1. Selected datasets from NeuroMorpho.Org

\begin{tabular}{|c|c|c|c|}
\hline Lab & Species & Region & Cell type \\
\hline Acsady & rat & ventral thalamus & modulated \\
\hline Alvarez & rat & spinal cord & motoneuron \\
\hline Amaral & rat & hippocampus & pyramidal \\
\hline Araujo & proechimys & hippocampus & pyramidal-like \\
\hline Araujo & rat & hippocampus & pyramidal \\
\hline Ascoli & mouse & spinal cord & motoneuron \\
\hline Ascoli & rat & basal forebrain & $\begin{array}{l}\text { choline acetyltransferase (ChAT)- } \\
\text { positive }\end{array}$ \\
\hline Ascoli & rat & basal forebrain & neuropeptide Y (NPY)-positive \\
\hline Ascoli & rat & hippocampus & not reported \\
\hline Avendano & rat & brainstem & Intersubnuclear neuron \\
\hline Barrionuevo & rat & hippocampus & pyramidal \\
\hline Bartos & mouse & hippocampus & basket \\
\hline Bartos & mouse & hippocampus & dendritic targeting \\
\hline Bartos & mouse & hippocampus & perisomatic targeting \\
\hline Bianchi & chimpanzee & neocortex & pyramidal \\
\hline Bikson & rat & neocortex & not reported \\
\hline Bikson & rat & neocortex & pyramidal \\
\hline Blackman & mouse & neocortex & basket \\
\hline Blackman & mouse & neocortex & pyramidal \\
\hline Brown & rat & neocortex & multipolar \\
\hline Brown & rat & neocortex & neurogliaform \\
\hline Brown & rat & neocortex & pyramidal \\
\hline Brown & rat & neocortex & tripolar \\
\hline Brumberg & mouse & neocortex & pyramidal \\
\hline Burke & cat & spinal cord & motoneuron \\
\hline Cameron & cat & spinal cord & motoneuron \\
\hline Cameron & rat & brainstem & motoneuron \\
\hline Cauli & rat & neocortex & neuropeptide Y (NPY)-positive \\
\hline Cauli & rat & neocortex & bipolar \\
\hline Cauli & rat & neocortex & pyramidal \\
\hline Chalupa & mouse & retina & ganglion \\
\hline Chmykhova & frog & spinal cord & motoneuron \\
\hline Chmykhova & turtle & spinal cord & motoneuron \\
\hline Cho & mouse & hippocampus & granule \\
\hline Claiborne & rat & hippocampus & granule \\
\hline Claiborne & rat & hippocampus & pyramidal \\
\hline Collin & pouched lamprey & retina & ganglion \\
\hline Cossart-Bernard & rat & hippocampus & oriens-lacunosum moleculare \\
\hline Cossart-Bernard & rat & hippocampus & perforant pathway-associated \\
\hline Cossart-Bernard & rat & hippocampus & perisomatic targeting \\
\hline Cossart-Bernard & rat & hippocampus & Schaffer-collateral associated \\
\hline Cossart-Bernard & rat & hippocampus & trilaminar \\
\hline
\end{tabular}




\begin{tabular}{|c|c|c|c|}
\hline$\overline{L a b}$ & Species & Region & Cell type \\
\hline Cox & $\begin{array}{l}\text { drosophila } \\
\text { melanogaster }\end{array}$ & $\begin{array}{l}\text { peripheral nervous } \\
\text { system }\end{array}$ & $\begin{array}{l}\text { multidendritic-dendritic ar- } \\
\text { borization (DA) }\end{array}$ \\
\hline De Koninck & rat & neocortex & pyramidal \\
\hline Del Negro & mouse & myelencephalon & non-glutamatergic \\
\hline Dendritica & guinea pig & cerebellum & Purkinje \\
\hline Dendritica & rat & basal ganglia & dopaminergic \\
\hline Dendritica & rat & cerebellum & Purkinje \\
\hline Dendritica & rat & neocortex & pyramidal \\
\hline Destexhe & cat & neocortex & pyramidal \\
\hline Destexhe & rat & dorsal thalamus & thalamocortical \\
\hline Dusart & mouse & cerebellum & Purkinje \\
\hline Esclapez & rat & hippocampus & pyramidal \\
\hline Feldmeyer & rat & neocortex & fast-spiking \\
\hline Feldmeyer & rat & neocortex & horizontal \\
\hline Feldmeyer & rat & neocortex & inverted \\
\hline Feldmeyer & rat & neocortex & multipolar \\
\hline Feldmeyer & rat & neocortex & pyramidal \\
\hline Feldmeyer & rat & neocortex & tangential \\
\hline Franca & rat & neocortex & nitrergic \\
\hline Fukunaga & mouse & main olfactory bulb & mitral \\
\hline Fukunaga & mouse & main olfactory bulb & tufted \\
\hline Fyffe & cat & spinal cord & Ia inhibitory \\
\hline Fyffe & cat & spinal cord & motoneuron \\
\hline Fyffe & cat & spinal cord & Renshaw \\
\hline Fyffe & cat & spinal cord & spinocerebellar \\
\hline Garcia-Cairasco & rat & hippocampus & granule \\
\hline Gonzalez-Burgos & monkey & neocortex & basket \\
\hline Gonzalez-Burgos & monkey & neocortex & double bouquet \\
\hline Gonzalez-Burgos & monkey & neocortex & neurogliaform \\
\hline Gonzalez-Burgos & monkey & neocortex & pyramidal \\
\hline Gonzalez-Burgos & mouse & neocortex & basket \\
\hline Gonzalez-Burgos & mouse & neocortex & pyramidal \\
\hline Groen & rat & hippocampus & pyramidal \\
\hline Gulyas & rat & hippocampus & calbindin (CB)-positive \\
\hline Gulyas & rat & hippocampus & cholecystokinin (CCK)-positive \\
\hline Gulyas & rat & hippocampus & calretinin (CR)-positive \\
\hline Gulyas & rat & hippocampus & pyramidal \\
\hline Gulyas & rat & hippocampus & parvalbumin (PV)-positive \\
\hline Hajos & mouse & hippocampus & Chandelier \\
\hline Halnes & mouse & thalamus & GABAergic \\
\hline Hay & rat & neocortex & pyramidal \\
\hline Helmstaedter & rat & neocortex & not reported \\
\hline Helmstaedter & rat & neocortex & pyramidal \\
\hline Henckens & rat & amygdala & pyramidal \\
\hline Henckens & rat & amygdala & stellate \\
\hline Henckens & rat & hippocampus & pyramidal \\
\hline
\end{tabular}




\begin{tabular}{|c|c|c|c|}
\hline Lab & Species & Region & Cell type \\
\hline Henckens & rat & neocortex & pyramidal \\
\hline Henny & rat & basal ganglia & dopaminergic \\
\hline Irintchev & rat & brainstem & motoneuron \\
\hline Jacobs & bottlenose dolphin & neocortex & aspiny \\
\hline Jacobs & bottlenose dolphin & neocortex & pyramidal-like \\
\hline Jacobs & chimpanzee & cerebellum & basket \\
\hline Jacobs & chimpanzee & cerebellum & Golgi \\
\hline Jacobs & chimpanzee & cerebellum & granule \\
\hline Jacobs & chimpanzee & cerebellum & Lugaro \\
\hline Jacobs & chimpanzee & cerebellum & stellate \\
\hline Jacobs & clouded leopard & cerebellum & basket \\
\hline Jacobs & clouded leopard & cerebellum & granule \\
\hline Jacobs & clouded leopard & cerebellum & Lugaro \\
\hline Jacobs & clouded leopard & cerebellum & stellate \\
\hline Jacobs & elephant & cerebellum & basket \\
\hline Jacobs & elephant & cerebellum & Golgi \\
\hline Jacobs & elephant & cerebellum & Lugaro \\
\hline Jacobs & elephant & cerebellum & stellate \\
\hline Jacobs & giraffe & cerebellum & basket \\
\hline Jacobs & giraffe & cerebellum & Golgi \\
\hline Jacobs & giraffe & cerebellum & granule \\
\hline Jacobs & giraffe & cerebellum & Lugaro \\
\hline Jacobs & giraffe & cerebellum & stellate \\
\hline Jacobs & giraffe & neocortex & crab-like \\
\hline Jacobs & giraffe & neocortex & neurogliaform \\
\hline Jacobs & giraffe & neocortex & pyramidal \\
\hline Jacobs & human & cerebellum & basket \\
\hline Jacobs & human & cerebellum & Golgi \\
\hline Jacobs & human & cerebellum & granule \\
\hline Jacobs & human & cerebellum & Lugaro \\
\hline Jacobs & human & cerebellum & stellate \\
\hline Jacobs & human & neocortex & pyramidal \\
\hline Jacobs & humpback whale & cerebellum & basket \\
\hline Jacobs & humpback whale & cerebellum & Golgi \\
\hline Jacobs & humpback whale & cerebellum & granule \\
\hline Jacobs & humpback whale & cerebellum & Lugaro \\
\hline Jacobs & humpback whale & cerebellum & stellate \\
\hline Jacobs & humpback whale & neocortex & aspiny \\
\hline Jacobs & humpback whale & neocortex & pyramidal-like \\
\hline Jacobs & humpback whale & neocortex & sternzelle \\
\hline Jacobs & manatee & cerebellum & basket \\
\hline Jacobs & manatee & cerebellum & stellate \\
\hline Jacobs & minke whale & neocortex & aspiny \\
\hline Jacobs & minke whale & neocortex & pyramidal \\
\hline Jacobs & minke whale & neocortex & pyramidal-like \\
\hline
\end{tabular}




\begin{tabular}{|c|c|c|c|}
\hline$\overline{\text { Lab }}$ & Species & Region & Cell type \\
\hline Jacobs & Siberian tiger & cerebellum & basket \\
\hline Jacobs & Siberian tiger & cerebellum & Golgi \\
\hline Jacobs & Siberian tiger & cerebellum & granule \\
\hline Jacobs & Siberian tiger & cerebellum & Lugaro \\
\hline Jacobs & Siberian tiger & cerebellum & stellate \\
\hline Jaeger & rat & basal ganglia & not reported \\
\hline Jaeger & rat & cerebellum & glutamatergic \\
\hline Jaffe & rat & hippocampus & not reported \\
\hline Jaffe & rat & hippocampus & pyramidal \\
\hline Johnson & domestic pig & hippocampus & granule \\
\hline Johnston & rat & hippocampus & pyramidal \\
\hline Jonas & rat & hippocampus & basket \\
\hline Kim & mouse & hippocampus & pyramidal \\
\hline Kisvarday & cat & neocortex & pyramidal \\
\hline Kole & rat & hippocampus & pyramidal \\
\hline Korngreen & rat & neocortex & pyramidal \\
\hline Krieger & mouse & neocortex & pyramidal \\
\hline Kubota & rat & neocortex & basket \\
\hline Lai & mouse & basal ganglia & medium spiny \\
\hline Lee & mouse & amygdala & pyramidal \\
\hline Lee & mouse & hippocampus & granule \\
\hline Lien & rat & hippocampus & dendritic targeting \\
\hline Lien & rat & hippocampus & perisomatic targeting \\
\hline Luebke & monkey & neocortex & pyramidal \\
\hline Luzzati & guinea pig & basal ganglia & Neuroblast \\
\hline Luzzati & mouse & basal ganglia & Neuroblast \\
\hline Mailly & rat & basal ganglia & dopaminergic \\
\hline Markram & rat & neocortex & basket \\
\hline Markram & rat & neocortex & bipolar \\
\hline Markram & rat & neocortex & bitufted \\
\hline Markram & rat & neocortex & Chandelier \\
\hline Markram & rat & neocortex & Descending \\
\hline Markram & rat & neocortex & double bouquet \\
\hline Markram & rat & neocortex & horizontal \\
\hline Markram & rat & neocortex & Martinotti \\
\hline Markram & rat & neocortex & neurogliaform \\
\hline Markram & rat & neocortex & not reported \\
\hline Markram & rat & neocortex & pyramidal \\
\hline Markram & rat & neocortex & Small \\
\hline Markram & rat & neocortex & stellate \\
\hline Martone & mouse & cerebellum & Purkinje \\
\hline Maxwell & cat & spinal cord & spinocerebellar \\
\hline Meyer & rat & neocortex & pyramidal \\
\hline Meyer & rat & neocortex & stellate \\
\hline Miller & salamander & retina & ganglion \\
\hline
\end{tabular}




\begin{tabular}{|c|c|c|c|}
\hline Lab & Species & Region & Cell type \\
\hline Mizrahi & mouse & main olfactory bulb & periglomerular \\
\hline $\begin{array}{l}\text { Mustaparta- } \\
\text { Lofaldli }\end{array}$ & moth & antennal lobe & olfactory \\
\hline Nolan & mouse & entorhinal cortex & stellate \\
\hline Nusser & rat & main olfactory bulb & deep short axon \\
\hline Nusser & rat & main olfactory bulb & external tufted cell (ETC) \\
\hline OpenWorm & C. elegans & $\begin{array}{ll}\text { pharyngeal } & \text { ner- } \\
\text { vous system } & \\
\end{array}$ & motoneuron \\
\hline OpenWorm & C. elegans & $\begin{array}{ll}\text { pharyngeal } & \text { ner- } \\
\text { vous system } & \end{array}$ & pharyngeal \\
\hline OpenWorm & C. elegans & $\begin{array}{ll}\text { somatic } & \text { nervous } \\
\text { system } & \end{array}$ & amphid \\
\hline OpenWorm & C. elegans & $\begin{array}{ll}\text { somatic } & \text { nervous } \\
\text { system } & \end{array}$ & motoneuron \\
\hline OpenWorm & C. elegans & $\begin{array}{ll}\text { somatic } & \text { nervous } \\
\text { system } & \end{array}$ & not reported \\
\hline OpenWorm & C. elegans & $\begin{array}{ll}\text { somatic } & \text { nervous } \\
\text { system } & \end{array}$ & ring \\
\hline OpenWorm & C. elegans & $\begin{array}{ll}\text { somatic } & \text { nervous } \\
\text { system }\end{array}$ & somatic \\
\hline Poorthuis & mouse & neocortex & pyramidal \\
\hline Poria & mouse & retina & ganglion \\
\hline Povysheva & rat & neocortex & not reported \\
\hline Rhode & cat & brainstem & vertical \\
\hline Rose & cat & spinal cord & motoneuron \\
\hline Santhakumar & rat & hippocampus & semilunar granule \\
\hline Sjostrom & mouse & neocortex & basket \\
\hline Sjostrom & mouse & neocortex & Martinotti \\
\hline Sjostrom & mouse & neocortex & pyramidal \\
\hline Smith & rat & ventral striatum & aspiny \\
\hline Smith & rat & ventral striatum & medium spiny \\
\hline Smith-Koizumi & rat & myelencephalon & inspiratory \\
\hline Soltesz & mouse & hippocampus & pyramidal \\
\hline Somogyi & rat & hippocampus & basket \\
\hline Spruston & rat & hippocampus & not reported \\
\hline Spruston & rat & hippocampus & pyramidal \\
\hline Staiger & rat & neocortex & pyramidal \\
\hline Staiger & rat & neocortex & stellate \\
\hline Strettoi & mouse & retina & ganglion \\
\hline Svoboda & rat & neocortex & pyramidal \\
\hline Sztarker & locust & optic Lobe & somatic \\
\hline Tepper & mouse & basal ganglia & tyrosine-hydroxylase-positive \\
\hline Timofeev & cat & neocortex & pyramidal \\
\hline Timofeev & cat & ventral thalamus & thalamocortical \\
\hline Todd & rat & spinal cord & projection neuron \\
\hline Turner & rat & hippocampus & dendritic targeting \\
\hline
\end{tabular}


bioRxiv preprint doi: https://doi.org/10.1101/787911: this version posted October 1, 2019. The copyright holder for this preprint (which was not certified by peer review) is the author/funder, who has granted bioRxiv a license to display the preprint in perpetuity. It is made available under aCC-BY-NC-ND 4.0 International license.

Dendritic constancy

Cuntz et al.

\begin{tabular}{|l|l|l|l|}
\hline Lab & Species & Region & Cell type \\
\hline Turner & rat & hippocampus & granule \\
\hline Turner & rat & hippocampus & pyramidal \\
\hline Turner & rat & hippocampus & total molecular layer projecting \\
\hline Vervaeke & mouse & cerebellum & Golgi \\
\hline Vuksic & mouse & hippocampus & granule \\
\hline Wearne-Hof & monkey & neocortex & pyramidal \\
\hline Wittner & guinea pig & hippocampus & pyramidal \\
\hline Zaitsev & monkey & neocortex & pyramidal \\
\hline
\end{tabular}

